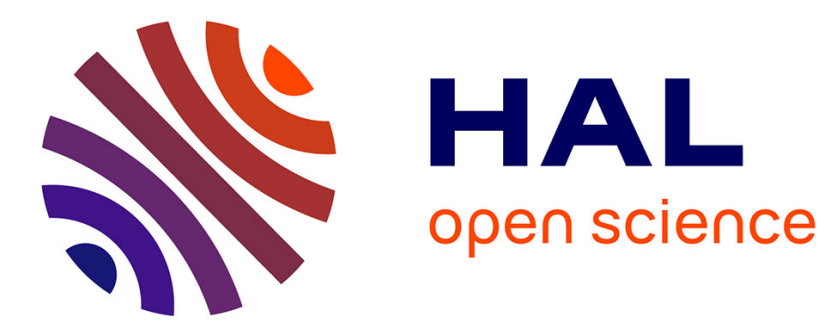

\title{
Flows on rooted trees and the Narayana idempotents
}

Frédéric Chapoton

\section{To cite this version:}

Frédéric Chapoton. Flows on rooted trees and the Narayana idempotents. Mathematica Scandinavica, 2014, 10.7146/math.scand.a-18001 . hal-00677425

\section{HAL Id: hal-00677425 \\ https://hal.science/hal-00677425}

Submitted on 8 Mar 2012

HAL is a multi-disciplinary open access archive for the deposit and dissemination of scientific research documents, whether they are published or not. The documents may come from teaching and research institutions in France or abroad, or from public or private research centers.
L'archive ouverte pluridisciplinaire $\mathbf{H A L}$, est destinée au dépôt et à la diffusion de documents scientifiques de niveau recherche, publiés ou non, émanant des établissements d'enseignement et de recherche français ou étrangers, des laboratoires publics ou privés. 


\title{
Flows on rooted trees and the Narayana idempotents
}

\author{
F. Chapoton
}

March 8, 2012

\begin{abstract}
Several generating series for flows on rooted trees are introduced, as elements in the group of series associated with the Pre-Lie operad. By combinatorial arguments, one proves identities that characterise these series. One then gives a complete description of the image of these series in the group of series associated with the Dendriform operad. This allows to recover the Lie idempotents in the descent algebras recently introduced by Menous, Novelli and Thibon. Moreover, one defines new Lie idempotents and conjecture the existence of some others.
\end{abstract}

\section{Introduction}

Let us start by introducing the context of this work, that can summarized by the following diagram.

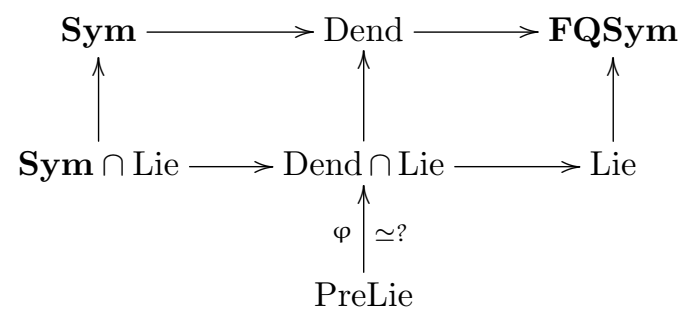

At the top left corner, Sym is the graded Hopf algebra of non-commutative symmetric functions [12], which has a basis indexed by compositions of integers. At the top right corner, FQSym is the graded Hopf algebra of free quasi-symmetric functions, also known as the Malvenuto-Reutenauer algebra [18], which has a basis indexed by permutations. These two Hopf algebras can be considered as non-commutative analogues of the classical Hopf algebra of symmetric functions. They have been studied a lot, and have proved to be useful in algebraic combinatorics, see for example [24, 8].

At the middle of the top line, Dend is the free Dendriform algebra on one generator. This is also a graded Hopf algebra, also known as the Loday-Ronco Hopf algebra [17], and has a basis indexed by planar binary trees. The horizontal morphisms of the first line are inclusions of Hopf algebras, and can be described using appropriate equivalence relations on permutations, see for instance [17]. 
On the second line, the subspace Lie of FQSym has two equivalent descriptions. First, one can map FQSym into a space of rational moulds, as described in [4]. Then Lie is the subspace of alternal elements, in the terminology of the mould calculus of Ecalle [10,9]. One can also identify FQSym with the direct sum of all group rings of symmetric groups, and therefore to the associative operad. Then Lie is the space of Lie elements, or the image of the Lie operad in the associative operad.

On the left of second line is the intersection of the subspaces Sym and Lie of FQSym. It is known to be exactly the subspace of primitive elements in the Hopf algebra Sym, by results of [12].

The intersection at the middle of the second line is quite interesting. Starting from the usual injective morphism from the Pre-Lie operad to the Dendriform operad, one gets an injective morphism $\varphi$ from the free Pre-Lie algebra on one generator, denoted here by PreLie, to Dend. It was proved in [4] that its image is contained in the intersection Dend $\cap$ Lie.

It is conjectured that $\varphi$ is an isomorphism from PreLie to Dend $\cap$ Lie. This has been checked for small degrees. If this isomorphism holds, it would have interesting consequences for the theory of Lie idempotents, that we will now present.

Recall that a Lie idempotent is an element $\theta$ in the group ring $\mathbb{Q}\left[\mathfrak{S}_{n}\right]$ of the symmetric group, such that $\theta$ is idempotent, and such that the product by $\theta$ is a projector onto the subspace of Lie elements. The set of Lie idempotents is an affine subspace of the group ring $\mathbb{Q}\left[\mathfrak{S}_{n}\right]$. There are many known examples of Lie idempotents, and most of them belong to a sub-algebra of $\mathbb{Q}\left[\mathfrak{S}_{n}\right]$, the Solomon descent algebra.

There is a natural way to identify $\mathbb{Q}\left[\mathfrak{S}_{n}\right]$ with the graded component of degree $\boldsymbol{n}$ of FQSym. By this isomorphism, Solomon descent algebra is identified with the graded component of degree $n$ of Sym. Moreover, the subspace of primitive elements of Sym corresponds to the intersection of Solomon descent algebras with the vector space spanned by Lie idempotents [12].

From all this, one can deduce that any Lie idempotent in the descent algebra gives an element in the intersection Sym $\cap$ Lie and therefore also in Dend $\cap$ Lie. If $\varphi$ is an isomorphism, this element will come from an element of PreLie. Conversely, given an element of PreLie, if one can check that its image by $\varphi$ belongs to $\mathbf{S y m}$, then it will belong to $\mathbf{S y m} \cap$ Lie and will define, up to multiplication by a scalar, a Lie idempotent in the descent algebra.

Given any specific Lie idempotent in the descent algebra, one can therefore ask for a description of its pre-image by $\varphi$. This has been obtained in [5] for a one parameter familly of Lie idempotents. The starting point of this article was to do the same for a specific familly of Lie idempotents, that has just been recently introduced. Let us now present them briefly.

Inspired by previous works by Ecalle and Menous [19, 11] on the Alien calculus, Menous, Novelli and Thibon have defined in [20] a sequence of Lie idempotents $D_{n}$ in the descent algebra of the symmetric group $\mathfrak{S}_{n}$. The coefficients of $D_{n}$ in the basis of ribbon Schur functions are given by homogeneous polynomials in two variables $a$ and $b$, more precisely products of powers of $a$ and $b$ and Narayana polynomials in $a$ and $b$. By homogeneity, one can let $a=1$ in the coefficients of $D_{n}$ without losing any information. We will therefore work with polynomials in $b$ only.

By computing, for small $n$, the elements $\mathcal{D}_{n}$ in PreLie whose image by $\varphi$ is 
$D_{n}$, one observes that their coefficients are positive polynomials in $b$ and seem to factorise according to subtrees, with factors being also positive polynomials in $b$.

The first result of the present article is a combinatorial description of the coefficients of $\mathcal{D}_{n}$ and their factors, in terms of flows on rooted trees.

To achieve this, one works inside groups of operadic series, associated with the Pre-Lie and Dendriform operads. All the idempotents $D_{n}$ are gathered into one series $D$ in the group $G_{\text {Dend }}$ associated with the Dendriform operad. Their pre-images $\mathcal{D}_{\mathfrak{n}}$ by $\varphi$ are similarly grouped in a series $\mathcal{D}$ in the group $G_{\text {PreLie }}$ associated with the Pre-Lie operad.

We proceed in the following order. First, we introduce the combinatorial notion of flow on a rooted tree, and describe its properties. Next, we obtain, from combinatorial arguments, various functional equations satisfied by several series in the Pre-Lie group, whose coefficients count different kinds of flows. We then go on to introduce some series in the Dendriform group, and to show, by algebraic means, that they satisfy another set of functional equations. By comparing the functional equations in the Pre-Lie and Dendriform cases, one can then recognize among the dendriform series the images by $\varphi$ of some of the Pre-Lie series.

On the way, one uses many auxiliary series, and some of them have interesting properties. In particular, one does not only recover the Lie idempotents $D_{n}$ of [20], but also gets a new familly $F_{n}$ of Lie idempotents, related to closed connected flows. Moreover, two other conjectural famillies $Z_{n}$ and $F_{n, t}$ of Lie idempotents are proposed, for which we have not been able to obtain a full proof. In the case of $F_{n, t}$, one is missing a combinatorial proof of the existence of a Pre-Lie series $\mathcal{F}_{t}$ and so we do not know if the dendriform series $F_{t}$ is a Lie element or not. In the case of $Z_{n}$, one only has a conjectural description of the coefficients of the dendriform series $Z$, and so we do not know if it belong to Sym.

We gather in an appendix some technical tools that are necessary to turn combinatorial bijections into equalities of series in groups associated with operads. The notions of rooted-operad and rooted-monoid that are introduced here may be of independent interest.

\section{Rooted trees and the PreLie operad}

\subsection{Notations for rooted trees}

A rooted tree is a finite connected and simply connected graph, together with a distinguished vertex called the root.

Rooted trees will be considered implicitly as directed graphs by orienting every edge towards the root.

The valency $v_{s}$ of a vertex $s$ in a rooted tree is the number of incoming edges.

The height of a vertex $s$ in a rooted tree is defined as follows: the height of the root is 0 , and the height of the source of every edge is 1 more than the height of its end.

Rooted trees of maximal height at most 1 are called corollas. Rooted trees of maximal valency at most 1 are called linear trees. 


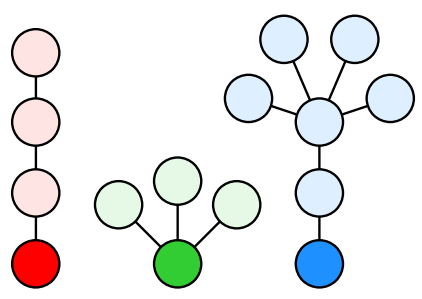

Figure 1: Linear tree $\mathrm{Lnr}_{4}$, corolla $\mathrm{Crl}_{3}$ and fork $\mathrm{Frk}_{3,4}$.

A rooted tree $T$ will sometimes be considered as a partially ordered set whose Hasse diagram is given by the orientation towards the root, with the root as the unique minimal element.

A leaf in a rooted tree $T$ is a vertex of valency 0 . A leaf can also be defined as a maximal vertex.

Rooted trees will be drawn with their root at the bottom and leaves at the top.

If $T_{1}, \ldots, T_{k}$ are rooted trees, we will denote $B_{+}\left(T_{1}, \ldots, T_{k}\right)$ the rooted tree obtained by grafting together $T_{1}, \ldots, T_{k}$ on a new common root.

Let $\bullet$ be the rooted tree with one vertex.

Let $\operatorname{Ln} r_{\ell}$ be the linear rooted tree with $\ell$ vertices, defined by induction:

$$
\operatorname{Ln} r_{1}=\bullet \quad \text { and } \operatorname{Ln} r_{\ell+1}=B_{+}\left(\operatorname{Ln} r_{\ell}\right) .
$$

Let $\operatorname{Cr} l_{n}$ be the corolla with $n+1$ vertices, defined by

$$
\mathrm{B}_{+}(\bullet, \ldots, \bullet)
$$

with $n$ copies of

Let $\operatorname{Frk}_{i, n-i}$ be the fork with $n$ vertices, with stem of size $i$, defined by induction:

$$
\operatorname{Frk}_{1, \ell}=\operatorname{Crl}_{\ell} \text { and } \operatorname{Frk}_{k+1, \ell}=B_{+}\left(\operatorname{Frk}_{k, \ell}\right) .
$$

Examples of linear trees, corollas and forks are depicted in figure 1.

The number of vertices of a rooted tree $\mathrm{T}$ will be denoted by \# $\mathrm{T}$.

\subsection{The group of rooted trees}

For more details on the general construction of the group of series associated $\mathrm{G}_{\mathcal{P}}$ with an operad $\mathcal{P}$, the reader may consult the appendix A, [7] and [5, App. A].

We will work in the group of series $G_{\text {PreLie }}$ associated with the Pre-Lie operad. This group is contained in the free Pre-Lie algebra on one generator, denoted here by PreLie.

The Pre-Lie operad has a basis indexed by labelled rooted trees [6]. It follows that the Pre-Lie algebra on one generator has a basis index by (unlabelled) rooted trees. 
For a series $\mathcal{D}$ in the group of rooted trees, we will use $\mathcal{D}_{\mathrm{T}}$ to denote the coefficient of the rooted tree $\mathrm{T}$ in $\mathcal{D}$, in the following sense:

$$
\mathcal{D}=\sum_{\mathrm{T}} \frac{\mathcal{D}_{\mathrm{T}}}{\operatorname{aut}(\mathrm{T})} \mathrm{T},
$$

where $\operatorname{aut}(\mathrm{T})$ is the cardinal of the automorphism group of $\mathrm{T}$.

The homogeneous component of $\mathcal{D}$ of degree $n$ will be denoted by $\mathcal{D}_{n}$.

We will use the following special notation for the sum of all corollas:

$$
\operatorname{CrLS}=\sum_{n \geq 0} \frac{\operatorname{Crl}_{n}}{n !} .
$$

Let $\mathrm{H}_{\mathrm{k}}$ be the element

$$
\mathrm{H}_{\mathrm{k}}=\sum_{\mathrm{T}} \mathrm{k}^{\# \mathrm{~T}-1} \frac{\mathrm{T}}{\mathrm{aut}_{\mathrm{T}}}
$$

of the group $\mathrm{G}_{\text {PreLie }}$ Its coefficients are polynomials in the variable $k$.

Lemma 1.1 One has

$$
\mathrm{H}_{\mathrm{k}} \circ \mathrm{H}_{\ell}=\mathrm{H}_{\mathrm{k}+\ell},
$$

where $\mathrm{k}$ and $\mathrm{\ell}$ are formal variables. In particular, when $\mathrm{k}$ is a positive integer,

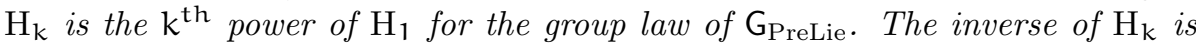
$\mathrm{H}_{-\mathrm{k}}$.

Proof. It is enough to prove this identity for $k$ and $\ell$ positive integers, by polynomiality.

Let $\mathrm{K}$ and $\mathrm{L}$ be finite sets of cardinality $\mathrm{k}$ and $\ell$. Elements of this sets are considered as colors.

One applies proposition A.5 for the rooted-operad PreLie, with $A$ the species of rooted trees with edges colored by elements of $\mathrm{K}, \mathrm{B}$ the species of rooted trees with edges colored by elements of $L$ and $C$ the species of rooted trees with edges colored by elements of $\mathrm{K} \sqcup \mathrm{L}$. The series $s_{A}, s_{B}$ and $s_{C}$ are clearly just $H_{k}, H_{\ell}$ and $\mathrm{H}_{\mathrm{k}+\ell}$.

The necessary bijection (hypothesis $H_{\sharp}(A, B, C)$ ) is obtained as follows. Pick any rooted tree $\mathrm{T}$ with edges colored by $\mathrm{K} \sqcup \mathrm{L}$. One considers the connected components in $\mathrm{T}$ with respect to the edges with color in $\mathrm{L}$. Each connected component is a rooted tree. Collapsing every connected component to a point, one obtains a rooted tree $\tau$ with edges colored by $\mathrm{K}$. To recover the original rooted tree $\mathrm{T}$, one has to know how to glue back the connected components into $\tau$. The different ways to do that are exactly counted by a constant of structure of the global composition map of the Pre-Lie operad.

The suspension $\Sigma$ is defined by

$$
\Sigma\left(\sum_{n \geq 1} a_{n}\right)=\sum_{n \geq 1}(-1)^{n-1} a_{n},
$$

where $a_{n}$ is homogeneous of degree $n$. 

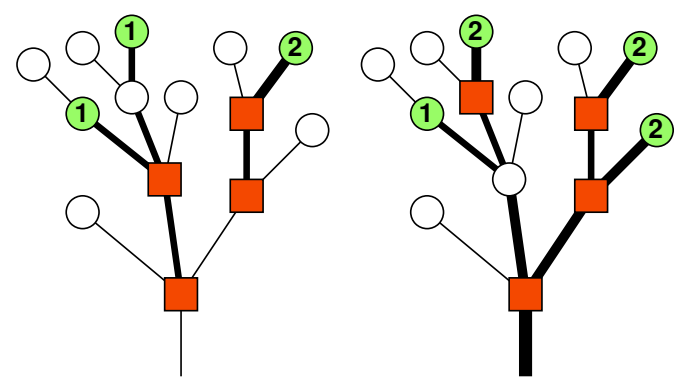

Figure 2: Two flows of size 4, on the same rooted tree with 14 vertices. Only the left one is closed.

\section{Combinatorics of flows}

\subsection{Definition}

Let $\mathrm{T}$ be a rooted tree. We will call a flow on $\mathrm{T}$ of size $\mathrm{k}$ the data of

- $\mathrm{k}$ distinct vertices of $\mathrm{T}$ (outputs),

- vertices of T (inputs), distinct from outputs, and that can be taken with multiplicities,

that has to satisfy the condition that we will introduce next.

Given inputs and $k$ outputs as above, one can define a rate in $\mathbb{Z}$ on every edge of $\mathrm{T}$ as follows.

- If the vertex $v$ is neither an input nor an output, the sum of incoming rates in $v$ is equal to the outgoing rate of $v$.

- If the vertex $v$ is an input with multiplicity $\ell$, the outgoing rate of $v$ is the sum of incoming rates in $v$ plus $\ell$.

- If the vertex $v$ is an output, the outgoing rate of $v$ is the sum of incoming rates in $v$ minus 1 .

The main requirement is that all rates are in $\mathbb{N}$.

Note that, by convention, the incoming rate in leaves is 0 , but the outgoing rate at the root (exit rate) can be an arbitrary positive integer.

If the exit rate is 0 , the flow is closed.

This definition is illustrated in Figure 2, where outputs are depicted by red squares and inputs by green circles - with their multiplicity. The rates, between 0 and 3, are drawn with increasing width.

Lemma 2.1 A closed flow of size $\mathrm{k}$ can also be described as

- $\mathrm{k}$ distinct vertices of $\mathrm{T}$ (outputs),

- $\mathrm{k}$ vertices of $\mathrm{T}$ (inputs), distinct from outputs, and that can be taken with multiplicities,

such that there exists $\mathrm{k}$ decreasing paths from one input to an output that make a one-to-one matching of inputs with outputs. 
Proof. Let us see why the data of a closed flow is equivalent to the existence of $k$ paths with the required properties.

Given $k$ decreasing paths matching inputs with outputs, one can find the rate of an edge by counting how many paths go through this edge. This rate function on edges does satisfy all the desired properties, and defines a closed flow.

Conversely, given a rate function on edges defining a closed flow, one can find paths, by induction on the size $k$. Let us pick an output and choose an increasing path of edges of strictly positive rate, until one reaches an input. This defines a path from the reached input to the chosen output. Removing this input and this output and subtracting 1 to the rate function for every edge of this path, one find another admissible rate function with $k$ decreased by 1 . Then by induction, one gets $k$ paths with the expected matching property.

Let $\mathbb{F}(\mathrm{T})$ be the set of flows on $T$ and $\mathbb{F}(T, k, \mathfrak{i})$ be the finite set of flows of size $k \in \mathbb{N}$ with exit rate $i \in \mathbb{N}$.

\subsection{Properties of closed flows}

Let us give some simple properties of the definition of closed flows.

For every rooted tree $T$, there is exactly one closed flow of size 0 , which is the empty flow, with no input vertex and no output vertex, where every edge has rate 0 .

For a rooted tree $\mathrm{T}$, closed flows of size 1 are in bijection with pairs of distinct comparable vertices of $T$. The number of closed flows of size 1 is therefore the sum of the heights of the vertices of $\mathrm{T}$.

Lemma 2.2 For a rooted tree $\mathrm{T}$, the maximal size of a flow on $\mathrm{T}$ is the number of non-leaf vertices of $\mathrm{T}$. There always exist a closed flow having this exact size.

Proof. Indeed, any output must be a non-leaf vertex, because it has to be smaller than an input vertex. Conversely, one can find a closed flow of this size by putting an output on every non-leaf vertex and inputs on leaves as follows. Going upwards in the tree, one can choose at each output where the incoming flow should come from, until one reaches leaves.

\subsection{Small flows}

Let us say that a flow $\psi \in \mathbb{F}(\mathrm{T})$ is small if the root is neither an output nor an input.

If the flow is closed, it is equivalent to require that the rate of every edge incoming in the root of $\mathrm{T}$ is 0 .

Denote by $\mathbb{F}^{s}(T)$ the set of small flows on $T$.

Lemma 2.3 If $\mathrm{T}=\mathrm{B}_{+}\left(\mathrm{T}_{1}, \ldots, \mathrm{T}_{\mathrm{k}}\right)$, there is a bijection

$$
\mathbb{F}^{s}(\mathrm{~T}) \simeq \prod_{i=1}^{k} \mathbb{F}\left(\mathrm{T}_{i}\right),
$$

where the factors are given by restriction of the flow to subtrees. 


\subsection{Inductive description of flows}

Let $\mathcal{E}_{\mathrm{T}, \mathrm{t}}$ be the generating function of flows on $\mathrm{T}$ with respect to size and exit rate:

$$
\mathcal{E}_{T, t}=\sum_{k, i \geq 0} \sum_{\psi \in \mathbb{F}(T, k, i)} b^{k} t^{i},
$$

and let $\mathcal{D}_{\mathrm{T}, \mathrm{t}}$ be the similar generating function of small flows on $\mathrm{T}$ :

$$
\mathcal{D}_{\mathrm{T}, \mathrm{t}}=\sum_{k, i \geq 0} \sum_{\psi \in \mathbb{F}^{s}(\mathrm{~T}, k, i)} b^{k} t^{i}
$$

Recall that Lemma 2.2 says in particular that the size of a flow on $\mathrm{T}$ is bounded by the number of non-leaf vertices of $T$. Therefore the generating functions $\mathcal{E}_{\mathrm{T}, \mathrm{t}}$ and $\mathcal{D}_{\mathrm{T}, \mathrm{t}}$ are polynomials in $\mathrm{b}$ with coefficients that are formal power series in $t$. We will see later that they are in fact polynomials in $b$ with coefficients that are rational functions in $t$.

By Lemma 2.2, the degree of $\mathcal{E}_{\mathrm{T}}$ as a polynomial in $\mathrm{b}$ is exactly the number of non-leaf vertices of $T$. The constant term of $\varepsilon_{T}$ with respect to $b$ is $1 /(1-t)^{\# T}$, because a flow without outputs is just the choice of how many inputs there are at every vertex.

For example, when $\mathrm{T}$ is the fork $\mathrm{Frk}_{2,2}$, one gets

$$
\varepsilon_{T, t}=\frac{1+5 b+3 b^{2}-t\left(9 b+8 b^{2}\right)+t^{2}\left(5 b+7 b^{2}\right)-t^{3}\left(b+2 b^{2}\right)}{(1-t)^{4}} .
$$

We will see later how to compute this by induction.

We will use the general convention that the value at $t=0$ of a series denoted by a symbol with index $t$ will be denoted by the same symbol without index $t$. For instance, let $\mathcal{E}_{\mathrm{T}}$ and $\mathcal{D}_{\mathrm{T}}$ be the value at $\mathrm{t}=0$ of $\mathcal{E}_{\mathrm{T}, \mathrm{t}}$ and $\mathcal{D}_{\mathrm{T}, \mathrm{t}}$.

Lemma 2.4 One has $\mathcal{D}_{\mathrm{B}_{+}\left(\mathrm{T}_{1}, \ldots, \mathrm{T}_{\mathrm{k}}\right), \mathrm{t}}=\prod_{i=1}^{\mathrm{k}} \mathcal{E}_{\mathrm{T}_{i}, \mathrm{t}}$.

Proof. This follows from the bijection of Lemma 2.3, and its simple behaviour with respect to size and exit rate.

We will now proceed to give an inductive description of the series $\mathcal{E}_{\mathrm{T}, \mathrm{t}}$ and $\mathcal{D}_{\mathrm{T}, \mathrm{t}}$.

Let $\mathrm{T}$ be a tree and $v \rightarrow u$ be an edge of $\mathrm{T}$, with $u$ closer to the root. Let $\mathrm{T} \curvearrowleft_{v} w$ be the tree obtained by adding a new vertex $w$ on top of $v$. Let $\mathrm{T} \curvearrowleft_{u} w$ be the tree obtained by adding a new vertex $w$ on top of $u$. Let $S$ and $T_{1}, \ldots, T_{k}$ be the trees obtained from $T$ by removing the edges incoming in $v$. Here $S$ is the bottom tree (containing the root of $T$ ) and $T_{1}, \ldots, T_{k}$ are the top trees. This is illustrated in figure 3 .

Theorem 2.5 With the previous notations, one has the following equalities:

$$
\mathcal{E}_{\mathrm{T} \curvearrowleft_{\nu} w, \mathrm{t}}=\mathcal{E}_{\mathrm{T} \curvearrowleft_{u} w, \mathrm{t}}+\mathrm{b} \mathcal{E}_{\mathrm{S}, \mathrm{t}} \prod_{i=1}^{\mathrm{k}} \mathcal{E}_{\mathrm{T}_{\mathrm{i}}},
$$

and

$$
\mathcal{D}_{\mathrm{T} \curvearrowleft \nu, t}=\mathcal{D}_{\mathrm{T} \curvearrowleft_{u} w, \mathrm{t}}+\mathrm{b} \mathcal{D}_{\mathrm{S}, \mathrm{t}} \prod_{i=1}^{\mathrm{k}} \mathcal{E}_{\mathrm{T}_{\mathrm{i}}} .
$$



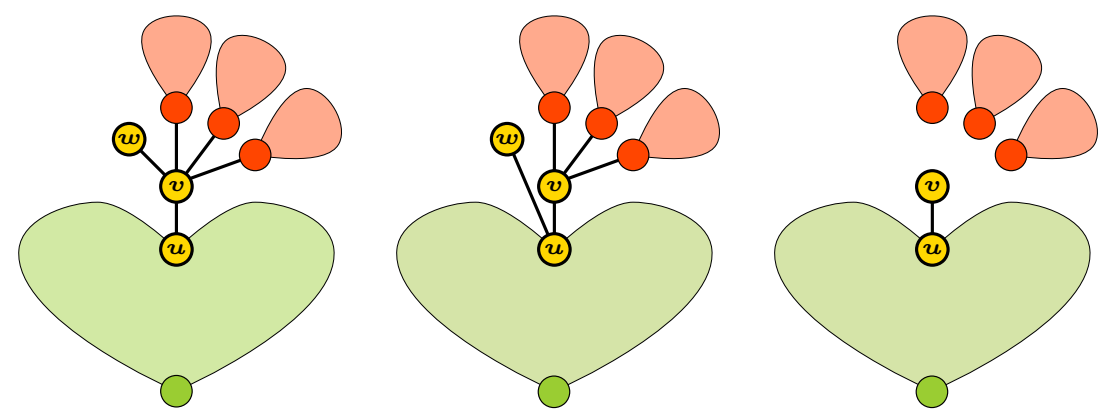

Figure 3: From left to right: $\mathrm{T} \curvearrowleft_{v} \mathcal{w}, \mathrm{T} \curvearrowleft_{\mathfrak{u}} w$ and $\mathrm{S}$ under $\mathrm{T}_{1}, \ldots, \mathrm{T}_{\mathrm{k}}$.

Proof. Let us prove the first equation.

Let us consider a flow on the tree $\mathrm{T} \curvearrowleft_{v} w$. Let $\alpha$ be the rate of $w \rightarrow v$ and $\beta$ be the rate of $v \rightarrow u$. One can distinguish two cases.

Either $\alpha=\beta+1$, in which case $v$ is an output, and all other edges incoming in $v$ have rate 0 . This kind of flow can be described in a bijective way using closed flows on the trees $T_{1}, \ldots, T_{k}$ and one flow on the tree $S$. This gives the rightmost term.

Otherwise $\alpha \leq \beta$. One can then define a flow on $T \curvearrowleft_{\mathfrak{u}} w$ as follows. One moves down the end of the edge $w \rightarrow v$ which becomes an edge $w \rightarrow u$ and keep the rate $\alpha$. The rate of the edge $u-v$ is set to $\beta-\alpha$ and remains positive. This clearly defines a bijection, and one gets the leftmost term.

Requiring in addition that the root is empty, the same proof gives the second identity.

The simplest case of this induction is when $v$ is a leaf in $\mathrm{T}$, in which case the rightmost term has just the factor associated with $S$.

This theorem can be used to compute $\mathcal{E}_{\mathrm{T}, \mathrm{t}}$ from smaller cases, by choosing a leaf $w$ of height at least 2. This is always possible, unless $\mathrm{T}$ is a corolla.

There is a nice commuting property to this induction. Indeed, one can use it in several different ways to compute $\mathcal{E}_{\mathrm{T}, \mathrm{t}}$, by choosing different leaves. This happens first for trees with 5 vertices.

One has the following consequence:

Corollary 2.6 Let $\mathrm{T}$ be a rooted tree. Then one has

$$
\mathcal{E}_{\mathrm{B}_{+}\left(\bullet, \mathrm{T}_{1}, \ldots, \mathrm{T}_{\mathrm{k}}\right), \mathrm{t}}=\frac{1}{1-\mathrm{t}}\left(\mathcal{E}_{\mathrm{B}_{+}\left(\mathrm{T}_{1}, \ldots, \mathrm{T}_{\mathrm{k}}\right), \mathrm{t}}+\mathrm{b} \prod_{i=1}^{\mathrm{k}} \mathcal{E}_{\mathrm{T}_{i}}\right) .
$$

Proof. This follows from equation (10). Indeed, one has

$$
\mathcal{D}_{\mathrm{B}_{+}\left(\mathrm{B}_{+}\left(\bullet, \mathrm{T}_{1}, \ldots, \mathrm{T}_{\mathrm{k}}\right)\right), \mathrm{t}}=\mathcal{D}_{\mathrm{B}_{+}\left(\bullet, \mathrm{B}_{+}\left(\mathrm{T}_{1}, \ldots, \mathrm{T}_{\mathrm{k}}\right)\right), \mathrm{t}}+\mathrm{b} \mathcal{D}_{\mathrm{B}_{+}(\bullet), \mathrm{t}} \prod_{i=1}^{\mathrm{k}} \mathcal{E}_{\mathrm{T}_{i}} .
$$

One can then use Lemma 2.4.

Corollary 2.6 can be used to compute the coefficients $\mathcal{E}_{\mathrm{Cr}_{n}, t}$ for corollas, by induction on $n$. 
Remark 2.7 When $\mathrm{t}=0$, Theorem 2.5 implies that the coefficients of $\mathcal{E}_{\mathrm{T}}$ (as a polynomial in $\mathrm{b}$ ) grow when a leaf is pulled up, as the rightmost term of (9) has positive coefficients.

\subsection{Properties of $\mathcal{E}_{\mathrm{T}}$}

Lemma 2.8 For every rooted tree $\mathrm{T}$, the series $\mathcal{E}_{\mathrm{T}, \mathrm{t}}$ is a polynomial in $\mathrm{b}$ of degree the number of non-leaf vertices of $\mathrm{T}$, with coefficients that are rational functions in $\mathrm{t}$, with poles only at $\mathrm{t}=1$. The common denominator of $\mathcal{E}_{\mathrm{T}, \mathrm{t}}$ is $(1-\mathrm{t})^{\# \mathrm{~T}}$.

Proof. The polynomial behaviour with respect to $b$ follows from the upper bound on the number of outputs, given by the number of non-leaf vertices, see Lemma 2.2. There always exists at least one flow with outputs at every non-leaf vertex, for example by placing sufficiently many inputs in every leaf. Therefore the degree of the polynomial is the number of non-leaf vertices.

Let us now show that the coefficients of this polynomial in $b$ are rational functions in $t$ with poles only at $t=1$ and of order at most the size of $T$. This

is true for the rooted tree $\bullet$, as $\mathcal{E}_{\bullet, t}=1 /(1-\mathrm{t})$. By corollary 2.6, this is true for all corollas, by induction. One can then use induction on the sum of heights of the vertices and on the number of vertices. Let $T$ be a tree which is not a corolla, and let $w$ be a leaf of maximal height in T. Take $v$ to be the vertex under $w$ and $u$ the vertex under $v$. Then one can apply Theorem 2.5 to prove the induction step.

It remains to show that the order of the pole at 1 of $\varepsilon_{T, t}$ is exactly the size of $\mathrm{T}$. This follows from the obvious fact that the constant term with respect to $\mathrm{b}$ is exactly $1 /(1-t)^{\# \mathrm{~T}}$.

The same kind of properties holds for $\mathcal{D}_{\mathrm{T}, \mathrm{t}}$, thanks to Lemma 2.4 and the obvious initial conditions $\mathcal{D}_{\mathrm{Cr}_{n}, \mathrm{t}}=1 /(1-\mathrm{t})^{\mathrm{n}}$.

\subsection{Connected flows}

Let us say that two vertices $u, v$ of $T$ are connected by the flow $\psi$ on $T$ if every edge of the unique path from $u$ to $v$ does have a strictly positive rate in $\psi$.

One can then define connected components with respect to the flow $\psi$, namely sets of vertices connected by the flow $\psi$. Each connected component with respect to a flow is a rooted tree.

A flow is called connected if it has exactly one connected component.

Let $\mathbb{F}^{\mathrm{c}}(\mathrm{T})$ be the set of connected flows on $\mathrm{T}$.

Lemma 2.9 If a rooted tree $\mathrm{T}$ admits a closed connected flow, its root has valency at most 1 .

Proof. The statement holds for the tree with one vertex. One can therefore assume that the tree $\mathrm{T}$ is not the trivial tree $\bullet$. By connectedness, every edge incident to the root contributes at least 1 to the total rate entering the root. By closure, the root is then necessarily an output, and it can only accept a rate of 1 . Therefore there is exactly one incident edge to the root.

We will consider now the question of what rooted trees admit a closed connected flow. 


\subsection{Trees with a closed connected flow}

We will now give a description of the rooted trees that admit a closed connected flow, using a function defined by Jean-Claude Arditti [1, 2] in relation to rooted trees with Hamiltonian comparability graphs. One can note that these references also use some kind of flows on rooted trees. To avoid possible confusion, we will call this function the valor, which is not the original terminology.

Let $T$ be a rooted tree. The valor $\mathbb{V}(f)$ of a leaf $f$ is 1 . The valor $\mathbb{V}(v)$ of a vertex $v$ is

$$
\max \left(1,-1+\sum_{s \rightarrow v} \mathbb{V}(s)\right)
$$

Lemma 2.10 The valor of the root of $\mathrm{T}$ is the minimal value of the exit rate among all connected flows on $\mathrm{T}$ with non-zero exit rate.

Proof. By induction on the size of the tree T. This is true for the tree $\bullet$, which has minimal non-zero exit rate 1 . Let $T=B_{+}\left(T_{1}, \ldots, T_{k}\right)$. Then the minimal exit rate of a connected flow on $\mathrm{T}$ is the sum of the minimal non-zero exit rates of $T_{1}, \ldots, T_{k}$, minus 1 corresponding to an output at the root of $T$. If this is at least 1 , this is the minimum non-zero exit rate. If this is zero, the minimum non-zero exit rate is 1 , and can be obtained by adding 1 to the rate along the path from the root to any chosen leaf.

This proves that the minimal non-zero exit rate satisfies the same recursion as the valor.

Proposition 2.11 A rooted tree $\mathrm{B}_{+}(\mathrm{T})$ admits a closed connected flow if and only if the root of $\mathrm{T}$ has valor 1 .

Proof. Using Lemma 2.9, the rooted tree $\mathrm{B}_{+}(\mathrm{T})$ admits a closed connected flow if and only if the rooted tree $T$ admits a connected flow with exit rate 1 . By the previous lemma, this is equivalent to say that the valor of the root of $T$ is 1 .

\subsection{Inductive description of connected flows}

Let us introduce a generating function for connected flows:

$$
\mathcal{E}_{T, t}^{c}=\sum_{k, i \geq 0} \sum_{\psi \in \mathbb{F}^{c}(T, k, i)} b^{k} t^{i}
$$

By Lemma 2.9, a rooted tree (different from $\bullet$ ) which admits a closed connected flow can be written $B_{+}(\mathrm{T})$. Let us denote by $\mathcal{F}_{\mathrm{T}}$ the generating series of connected flows on $\mathrm{T}$ with exit rate 1.

We will now obtain an inductive description of the coefficients $\mathcal{F}_{\mathrm{T}}$.

Let us consider the situation depicted in figure 4, with the same notations as for Theorem 2.5. The tree $S$ is obtained from $T$ by removing everything above $v$. The tree $S^{\prime}$ is obtained as the subtree of $T \curvearrowleft v w$ with root $v$.

Theorem 2.12 With the previous notations, one has the following equalities:

$$
\mathcal{F}_{\mathrm{T}{ }_{v} w}=\mathcal{F}_{\mathrm{T}{ }_{u} w}+\mathcal{F}_{\mathrm{S}} \mathcal{F}_{\mathrm{S}^{\prime}} \text {. }
$$



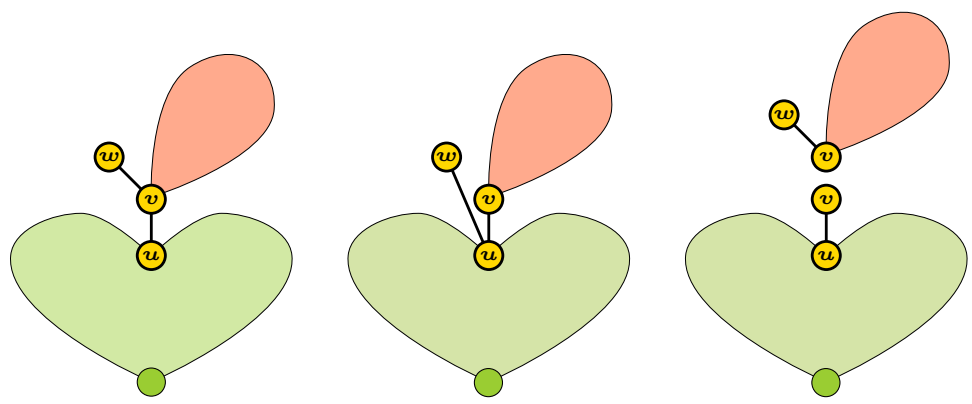

Figure 4: From left to right: $\mathrm{T} \curvearrowleft_{v} w, \mathrm{~T} \curvearrowleft_{\mathfrak{u}} w$ and $\mathrm{S}$ under $\mathrm{S}^{\prime}$.

Proof. Let us consider a connected flow on $T \curvearrowleft_{v} w$ with exit rate 1 . Let $\alpha \geq 1$ be the rate of the edge $w \rightarrow v$ and $\beta \geq 1$ be the rate of the edge $v \rightarrow u$.

If $\beta \geq \alpha+1$, then one can define a connected flow on $T \curvearrowleft_{\mathfrak{u}} \mathcal{w}$ with exit rate 1 as follows. One replaces the edge $w \rightarrow v$ by an edge $w \rightarrow u$ with rate $\alpha$, and assign the rate $\beta-\alpha \geq 1$ to the edge $v \rightarrow u$. This is clearly a bijection, and gives the leftmost term.

Otherwise, one has $\beta \leq \alpha$. One can then define a connected flow on $S^{\prime}$ with exit rate 1 and a connected flow on $S$ with exit rate 1 , as follows. On the bottom tree $S$, the vertex $v$ becomes an input with exit rate $\beta$, and all rates are unchanged. On the top tree $S^{\prime}$, the vertex $v$ has the same content as the vertex $v$ of $\mathrm{T} \curvearrowleft_{v} w$, either input or output. One assigns to the edge $w \rightarrow v$ the rate $\alpha-\beta+1 \geq 1$. One can check that the exit rate of this connected flow on $S^{\prime}$ is 1. This construction is clearly a bijection, and one obtains the rightmost term.

For example, one can compute using this theorem that $\mathcal{F}_{\mathrm{Frk}_{2,2}}$ is $2 \mathrm{~b}(1+\mathrm{b})$.

Corollary 2.13 For every rooted tree $\mathrm{T}$ with $\mathrm{n}$ vertices, the coefficient of $\mathrm{b}^{\mathrm{k}}$ and the coefficient of $\mathrm{b}^{\mathrm{n}-1-\mathrm{k}}$ in $\mathcal{F}_{\mathrm{T}}$ are equal.

Proof. This is certainly true for small corollas by inspection, and $\mathcal{F}_{\mathrm{Cr}_{n} \text { n }}$ vanishes if $n \geq 3$. Then one can proceed by induction on the size and the total height, using (14).

One may wonder whether this unexpected symmetry has a combinatorial description.

It appears that it may be possible to introduce a parameter $t$ in the inductive definition (14).

Conjecture 2.14 We keep the same notations as for Theorem 2.12. There exists rational functions $\mathcal{F}_{\mathrm{T}, \mathrm{t}}$, such that

$$
\mathcal{F}_{\mathrm{T} \curvearrowleft{ }_{v} w, t}=\mathcal{F}_{\mathrm{T} \curvearrowleft_{u} w, t}+(1-\mathrm{t}) \mathcal{F}_{\mathrm{S}, \mathrm{t}} \mathcal{F}_{\mathrm{S}^{\prime}, \mathrm{t}},
$$

and such that

$$
\mathcal{F}_{\operatorname{Lnr}(\mathrm{n}), \mathrm{t}}=\mathcal{E}_{\operatorname{Lnr}(\mathrm{n}), \mathrm{t}} \text { for } \mathrm{n} \geq 1 \text {, }
$$

and

$$
\mathcal{F}_{\mathrm{Cr}_{\mathrm{n}}}=\mathrm{b}(-\mathrm{t})^{\mathrm{n}-2} /(1-\mathrm{t})^{\mathrm{n}-1} \quad \text { for } \mathrm{n} \geq 2
$$




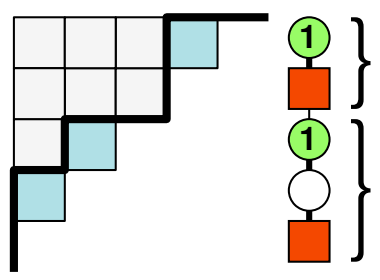

Figure 5: Bijection between Dyck paths and closed flows on linear trees. In this example, the flow has two connected components.

It is easy to prove that this defines uniquely the fractions $\mathcal{F}_{\mathrm{T}, \mathrm{t}}$, if they exist.

For example, one gets that

$$
\mathcal{F}_{\mathrm{Frk}_{2,2}, \mathrm{t}}=\frac{\mathrm{b}}{(1-\mathrm{t})^{2}}+\frac{\mathrm{b}(1+2 \mathrm{~b})}{1-\mathrm{t}} .
$$

Looking at the first fractions $\mathcal{F}_{\mathrm{T}, \mathrm{t}}$, one observes that they do not have positive coefficients as formal power series in $t$ and $b$, for example for the rooted tree

$\mathrm{B}_{+}\left(\mathrm{Crl}_{2}, \boldsymbol{\bullet}, \boldsymbol{\bullet}\right)$. They can therefore not be given a combinatorial description similar to the one for $\mathcal{F}_{\mathrm{T}}$ in terms of connected flows with exit rate 1 .

\subsection{Flows on linear trees and Dyck paths}

Let us consider the case of the linear trees. We first show that closed flows on linear trees are in bijection with very classical objects, namely Dyck paths.

Recall that a Dyck path of length $2 n$ is a plane lattice path from $(0,0)$ to $(n, n)$ using steps $(0,1)$ (up) and $(1,0)$ (right) and keeping above the diagonal line $y=x$. A Dyck path of length at least 2 is called indecomposable if it only touches the diagonal line at its extremities. Every Dyck path can by uniquely written as the concatenation of indecomposable Dyck paths. Every indecomposable Dyck path can be uniquely written $(0,1) \mathrm{D}(1,0)$ where $\mathrm{D}$ is a Dyck path. A peak in a Dyck path is a factor $(0,1)(1,0)$. We say that two letters $(0,1)$ and $(1,0)$ appearing in this order in a Dyck path are matched if the factor between them is a Dyck path.

Proposition 2.15 There exists a bijection $\rho$ between closed flows on $\mathrm{Ln}_{\mathrm{n}}$ and Dyck paths of length $2 \mathrm{n}$ through which

- connected components correspond to indecomposable factors,

- outputs correspond to matched pairs of steps that do not form a peak.

Proof. The bijection is defined by induction on $n$. If $n=1$, there is only one closed flow on $\bullet$, which has no output, and only one Dyck path, which is $(0,1)(1,0)$.

Assume now that $n$ is at least 2, and the bijection $\rho$ is defined for smaller $n$.

Any closed flow can be written as a list of connected components, starting from the component containing the root. Its image by $\rho$ is defined as the concatenation of the images by $\rho$ of the connected components. 
If there are at least 2 connected components, this defines $\rho$ by induction.

If not, the closed flow is connected. Then the root is an output. One can remove 1 to the rate of every edge and remove the root. This defines a closed flow on the linear tree with one vertex less. Its image by $\rho$ is taken to be $(0,1) \mathrm{D}(1,0)$, where $\mathrm{D}$ is the image by $\rho$ of the smaller flow, defined by induction.

This decomposition is obviously mapped to the similar classical decomposition of Dyck paths, using sub-Dyck paths and down-moving of indecomposable paths. The inverse bijection is immediate.

The statement on outputs follows easily by inspection of the bijection.

The bijection is illustrated in figure 5 . $\mathcal{E}_{\text {Lnr }_{n}}$.

Let $\mathbf{c a}_{n, t}$ be the generating series $\mathcal{E}_{\mathrm{Lnr}_{n}, t}$ and let $\mathbf{c a}_{n}$ be the polynomial

The first few values of $\mathbf{c a}_{\mathrm{n}, \mathrm{t}}$ are

$$
\begin{aligned}
& \mathbf{c a}_{1, t}=\frac{1}{1-t}, \quad \mathbf{c a}_{2, t}=\frac{1+b-t b}{(1-t)^{2}} \\
& \mathbf{c a}_{3, t}=\frac{1+3 b+b^{2}-t\left(4 b+2 b^{2}\right)+t^{2}\left(b+b^{2}\right)}{(1-t)^{3}}
\end{aligned}
$$

From the bijection above, it follows that ca $_{n}$ counts Dyck paths according to the number of peaks. These polynomials are classical in combinatorics, and known as the Narayana polynomials, see for example [13]. We will call $\mathbf{c a}_{n, t}$ a t-Narayana fraction.

Let us introduce ordinary generating series

$$
E=\sum_{n \geq 1} \mathbf{c a}_{n} x^{n} \text { and } E_{t}=\sum_{n \geq 1} \mathbf{c a}_{n, t} x^{n}
$$

and let $E^{c}$ be the similar series for closed connected flows on linear trees.

The analogous series for small flows are just $x(1+E)$ and $x\left(1+E_{t}\right)$, because a small flow on $\operatorname{Ln} r_{n+1}$ can be described by a flow on $\operatorname{Ln} r_{n}$.

From the combinatorial decomposition used in the bijection with Dyck paths, one deduces that

$$
E=E^{c} /\left(1-E^{c}\right) \text { and } E^{c}=x(1+b E) .
$$

By decomposing a flow according to whether the root is an output or not, one obtains the equation

$$
E_{t}=x /(1-t)\left(1+E_{t}\right)+b x / t\left(E_{t}-E\right) .
$$

This is a special case of the global equation for flows (23), that we will prove later.

It follows from all this that $E$ and $E^{c}$ are algebraic over $\mathbb{Q}(x)$ and that $E_{t}$ is algebraic over $\mathbb{Q}(x, t)$.

\subsection{Conjectural formula for closed flows on forks}

Recall from $\S 1.1$ that $\operatorname{Frk}_{i, n-i}$ is the fork with $n$ vertices, with stem of size $i$. 
Conjecture 2.16 The number of closed flows of size $\mathrm{k}$ on the fork $\mathrm{Frk}_{\mathrm{i}, \mathrm{n}-\mathrm{i}}$ is given by

$$
\# \mathbb{F}\left(\operatorname{Frk}_{i, n-i}, k, 0\right)=\left(\begin{array}{l}
i \\
k
\end{array}\right)\left(\begin{array}{l}
n \\
k
\end{array}\right)-\left(\begin{array}{l}
i+1 \\
k+1
\end{array}\right)\left(\begin{array}{l}
n-1 \\
k-1
\end{array}\right) .
$$

For $i=n-1$ or $i=n$, corresponding to linear trees, this formula gives the Narayana numbers, which is the correct result for the linear trees (see section 2.9). One can easily check that this also gives the correct answer for $i=1$, namely for corollas.

\subsection{Zeroes of flow polynomials}

After inspection of some examples, one is tempted to ask the following question.

Question 2.17 Let $\mathrm{T}$ be a rooted tree. Are the zeroes of $\mathcal{F}_{\mathrm{T}}$ real and negative ? Are the zeroes of $\mathcal{E}_{\mathrm{T}}$ real and negative?

It is known, for the Narayana polynomials, that all roots are real, simple and negative, see for example [13]. Therefore the question has a positive answer for linear trees. One can also check easily that this is true for corollas.

\section{Series of flows}

\subsection{Global equations for flows}

Let us introduce now two series

$$
\varepsilon_{\mathrm{t}}=\sum_{\mathrm{T}} \varepsilon_{\mathrm{T}, \mathrm{t}} \frac{\mathrm{T}}{\operatorname{aut}(\mathrm{T})} \quad \text { and } \quad \mathcal{D}_{\mathrm{t}}=\sum_{\mathrm{T}} \mathcal{D}_{\mathrm{T}, \mathrm{t}} \frac{\mathrm{T}}{\operatorname{aut}(\mathrm{T})},
$$

in the group $\mathrm{G}_{\text {PreLie }}$ associated with the Pre-Lie operad.

Let $\mathcal{E}($ resp. $\mathcal{D})$ be the value at $\mathrm{t}=0$ of $\mathcal{E}_{\mathrm{t}}\left(\right.$ resp. $\left.\mathcal{D}_{\mathrm{t}}\right)$.

Theorem 3.1 The following identity holds:

$$
\mathcal{D}_{\mathrm{t}}=\operatorname{CRLS} \diamond\left(\bullet, \varepsilon_{\mathrm{t}}\right) .
$$

Proof. This is essentially a restatement of Lemma 2.3, using the notation defined in (2) and the results of the appendix A.

Namely, one applies Prop. A.4 of the appendix, with $A$ the species of corollas, $B$ the species made only of the rooted tree on one vertex, $C$ the species of flows on rooted trees and $\mathrm{D}$ the species of small flows on rooted trees.

Theorem 3.2 One has

$$
\varepsilon_{\mathrm{t}}=\frac{1}{1-\mathrm{t}} \mathcal{D}_{\mathrm{t}}+\frac{\mathrm{b}}{\mathrm{t}}\left(\mathcal{D}_{\mathrm{t}}-\mathcal{D}\right) .
$$

Proof. Consider a rooted tree $T=B_{+}\left(T_{1}, \ldots, T_{k}\right)$ endowed with a flow.

Either the root is an input vertex with multiplicity $\ell$ for some $\ell \geq 0$. This can be described using a small flow and the integer $\ell$. One obtains

$$
\frac{1}{1-t} \mathcal{D}_{\mathrm{t}}
$$


The other possibility is that the root is an output vertex. Removing the output, one gets a small flow with the condition that the exit rate is not zero. This gives the term

$$
\frac{\mathrm{b}}{\mathrm{t}}\left(\mathcal{D}_{\mathrm{t}}-\mathcal{D}\right)
$$

\subsection{Global equations for connected flows}

Let $\varepsilon_{t}^{c}$ be the global series of connected flows:

$$
\mathcal{E}_{\mathrm{t}}^{\mathrm{c}}=\sum_{\mathrm{T}} \mathcal{E}_{\mathrm{T}, \mathrm{t}}^{\mathrm{c}} \frac{\mathrm{T}}{\operatorname{aut}(\mathrm{T})}
$$

and let $\mathcal{E}^{\mathrm{c}}$ be its value at $\mathrm{t}=0$.

Theorem 3.3 The series $\mathcal{E}_{\mathrm{t}}^{\mathrm{c}}$ satisfies the following equation

$$
\mathcal{E}_{\mathrm{t}}^{\mathrm{c}}=\frac{1}{1-\mathrm{t}} \operatorname{CRLS} \diamond\left(\bullet, \mathcal{E}_{\mathrm{t}}^{\mathrm{c}}-\mathcal{E}^{\mathrm{c}}\right)+\frac{\mathrm{b}}{\mathrm{t}}\left(\operatorname{CRLS} \diamond\left(\bullet, \mathcal{E}_{\mathrm{t}}^{\mathrm{c}}-\mathcal{E}^{\mathrm{c}}\right)-\bullet\right) .
$$

Proof. This is similar to the proof of Theorems 3.1 and 3.2. One has to distinguish according to the status of the root.

If the root is an input (possibly empty), the restriction to every subtree is an arbitrary connected flow with non-zero outgoing rate at the root. We obtain the first term of the right-hand side.

If the root is an output, there must be at least one subtree, and the restriction to every subtree is an arbitrary connected flow with non-zero outgoing rate at the root. This gives the second term of the right-hand-side.

The series $\mathcal{E}_{\mathrm{t}}$ of flows can be recovered from the series $\mathcal{E}_{t}^{c}$ of connected flows.

Theorem 3.4 There holds

$$
\varepsilon_{\mathrm{t}}=\left(\sum_{\mathrm{T}} \frac{\mathrm{T}}{\operatorname{aut}(\mathrm{T})}\right) \diamond\left(\mathcal{E}_{\mathrm{t}}^{\mathrm{c}}, \mathcal{E}^{\mathrm{c}}\right) .
$$

Proof. This follows from Prop. A.4 applied to the following four species: A is the species of rooted trees, B the species of connected flows, C the species of closed connected flows and $\mathrm{D}$ the species of flows.

The necessary bijection (hypothesis $\mathrm{H}_{\natural}(A, B, C, D)$ ) is rather clear. Indeed, given any flow, one can define connected flows on its connected components, closed if not containing the root. One can also make a rooted tree $\tau$ with vertices the connected components. To be able to recover the flow, one has to know how to glue back components into the tree $\tau$. This is given by a constant of structure of the global composition of the Pre-Lie operad.

In words, this theorem says that the series $\varepsilon_{t}$ of flows is obtained from the series of all trees, by insertion of $\mathcal{E}_{\mathrm{t}}^{\mathrm{c}}$ in the root and insertion of $\mathcal{E}^{\mathrm{c}}$ in all other vertices. 
When $t=0$, this reduces to the factorisation of series

$$
\mathcal{E}=\left(\sum_{\mathrm{T}} \frac{\mathrm{T}}{\operatorname{aut}(\mathrm{T})}\right) \circ \mathcal{E}^{\mathrm{c}},
$$

in the group $\mathrm{G}_{\text {PreLie }}$, which means that a closed flow is made by gluing closed connected flows along a rooted tree.

Because rooted trees that support closed connected flows have root-valency at most 1 by Lemma 2.9, one can write

$$
\mathcal{E}^{\mathrm{c}}=\bullet+\mathrm{b} \bullet \curvearrowleft \mathcal{F},
$$

for some series $\mathcal{F}$. We will use this series later on.

\subsection{Quotient series $\mathcal{E} \circ \mathcal{D}^{-1}$}

A saturated flow is a closed connected flow where every non-leaf vertex is an output.

Let $\mathcal{E}_{\mathrm{T}}^{\mathrm{s}}$ be the generating series for saturated flows on $T$. Note that this is a monomial in the variable $b$, of degree the number of non-leaf vertices of $T$.

Lemma 3.5 Let $\mathrm{T}$ be a rooted tree that admits a closed connected flow. Then $\mathrm{T}$ admits a saturated flow.

Proof. Pick a closed connected flow on T. The proof is by induction on the number of non-leaf vertices which are not outputs. If the chosen flow is saturated, there is nothing to do. Otherwise, let $v$ be a non-leaf vertex which is not an output.

If $v$ is not an input, one can put an output in $v$, choose a path from $v$ to some leaf $w$ of the subtree at $v$, and add 1 to the rate on every edge of this path and 1 input on $w$.

If $v$ is an input, one can first move this input to a leaf, by choosing a path from $v$ to a leaf $w$ of the subtree at $v$, and adding 1 to the rate on every edge of this path. Then one gets back to the previous case.

Therefore rooted trees that admit closed connected flows are exactly the same as rooted trees that admit saturated flows.

Let now $y$ be the quotient series $\mathcal{E} \circ \mathcal{D}^{-1}$ in the group $G_{\text {PreLie. }}$ One observes a surprising property.

Conjecture 3.6 The coefficient $y_{\mathrm{T}}$ of a rooted tree $\mathrm{T}$ in $\mathrm{y}$ is the monomial

$$
(-1)^{\mathrm{L}(\mathrm{T})-1} \mathcal{E}_{\mathrm{T}}^{\mathrm{s}}
$$

where $\mathrm{L}(\mathrm{T})$ is the number of leaves of $\mathrm{T}$.

If this is true, then by Lemma 3.5, the support of $y$ is the same as the support of $\mathcal{E}^{c}$, and one can write

$$
y=\bullet+b \bullet \curvearrowleft z,
$$

for some series $z$. We will consider this series again later. 

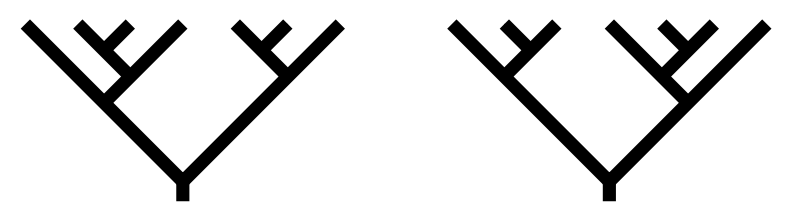

Figure 6: A planar binary tree with 6 inner vertices, and its image by reversal.

\section{Planar binary trees, dendriform operad and Sym}

\subsection{Notations for planar binary trees}

A planar binary tree on $n$ vertices is either the tree $1=\mid$ with no inner vertex or a pair of two planar binary trees. Planar binary trees will be drawn with their root at the bottom and leaves at the top, aligned on a horizontal line. Examples are depicted in figure 6.

There is a natural involution on the set of planar binary trees, given by left-right reversal, as shown in figure 6 .

The canopy of a planar binary tree is a sequence of letters $\oslash$ and $\oslash$ of length $n-1$. There is a letter for each leaf but the leftmost and rightmost one. The letter is $\Theta$ is the leaf is the left son of its parent vertex, and $\oslash$ is the leaf is the right son of its parent vertex.

For example, the canopy of the planar binary tree at the left of of figure 6 is 00000 .

We will also use the following variants: the full canopy is obtained from the canopy by adding $\odot$ at the beginning and $\oslash$ at the end, the left-completed canopy by adding $\odot$ at the beginning, and the right-completed canopy by adding $\oslash$ at the end.

\subsection{The Dendriform operad}

The Dendriform operad, introduced by Loday, is a non-symmetric operad with a basis indexed by planar binary trees. The free dendriform algebra is just the direct sum of all components of the Dendriform operad. We refer the reader to [16] for more information on the dendriform algebras.

On the free dendriform algebra Dend, there are two dendriform products $\prec$ and $\succ$, that satisfy the 3 dendriform axioms. In particular, their sum defines an associative product

$$
x * y=x \succ y+x \prec y,
$$

which is the product used in the Hopf algebra structure of Dend.

We will use the following notation:

$$
x \vee_{y} z=x \succ y \prec z .
$$

By one of the dendriform axioms, no parentheses are needed in this expression. When $y$ is the planar binary tree $Y$, the operation $x \vee z$ can be described as the gluing of $x$ and $z$ on a common vertex. 
From the dendriform axioms, one can deduce the following relations :

$$
(x \vee y) \prec z=x \vee(y * z) \text { and } x \succ(y \vee z)=(x * y) \vee z
$$

Oen can extend (in a unique way) the notation $x \vee_{y} z$ to the cases where $x$ or $z$ are the unit tree 1 , with the same properties.

Let $\varphi$ be the operad morphism from the PreLie operad to the Dend operad defined by its value on the labelled generator:

$$
\varphi(x \curvearrowleft y)=y \succ x-x \prec y .
$$

Therefore, the map $\varphi$ sends ${ }$ to $Y-Y$. One can show that the morphism $\varphi$ is injective by using that it factorises through the Brace operad.

From now on, the expression "dendriform image" will mean the image by $\varphi$.

We will work in the group $G_{\text {Dend }}$ associated with the dendriform operad. This is an open subset in the free dendriform algebra on one generator Dend.

Lemma 4.1 Let $\mathrm{T}$ be a labeled rooted tree, with $\mathrm{i}$ the label of the root. The dendriform image of $\mathrm{T}$ is a linear combination of labelled planar binary trees whose root is labeled by $i$.

Proof. One has to show that $\varphi$ is a morphism of rooted-operads, in the language of the appendix A. This is clear on the generators by (34), hence one can apply Lemma A.1.

Lemma 4.2 Let $x, y, z, t$ in Dend. Then

$$
(x \vee y) \diamond(z, t)=(x \circ t) \vee_{z}(y \circ t)
$$

Proof. This is an easy consequence of the definition (32) of $\vee$ and of the general definition of the operations $\diamond$ and $\circ$ in appendix A.

Lemma 4.3 Let $x, y, z, t, u$ in Dend. Let $v=\left(y \vee_{z} t\right)$. Then

$$
x \vee_{v} u=(x * y) \vee_{z}(t * u)
$$

Proof. This is a simple computation in the dendriform operad, starting from the definition (32).

The suspension $\Sigma$ is defined by

$$
\Sigma\left(\sum_{n \geq 1} a_{n}\right)=\sum_{n \geq 1}(-1)^{n-1} a_{n},
$$

where $a_{n}$ is homogeneous of degree $n$.

We will also use the bar involution, which is the composition of suspension and reversal, that are two commuting involutions. 


\subsection{The subalgebra Sym of Dend}

Let Sym be the algebra of non-commutative symmetric functions. This is the free associative algebra generated by one generator in every positive degree. We will use the basis of ribbon Schur functions, indexed by compositions of $n$ in degree $n$. For more information, the reader may consult $[12,24,8]$.

Compositions of $n$ will be identified with strings of $n-1$ symbols $\oslash$ and $\Theta$, by the convention that a $\odot$ symbol means "cut here" and a $\oslash$ symbols means "do not cut here". For example,

$$
1 / 4|1| 2 \longleftrightarrow 0000000
$$

The product in the basis of ribbon Schur functions is given by the rule

$$
\epsilon * \delta=\epsilon \oslash \delta+\epsilon \bigcirc \delta
$$

The inclusion from Sym to Dend is defined on the basis of Schur function by sending a sequence of elements of $\{\oslash, \Theta\}$ to the sum of all planar binary having this sequence as canopy. This is a morphism of algebras.

One will need the following lemma.

Lemma 4.4 Let $\theta$ be a Lie idempotent in the descent algebra of $\mathfrak{S}_{n}$, seen as an element of Sym. Then the coefficient of the ribbon Schur function with index $\partial^{\mathrm{n}-1}$ in $\theta$ is $1 / \mathrm{n}$.

Proof. By [23, Prop. 2.4], the coefficient of Id in the expansion of any Lie idempotent in the usual basis of the symmetric group ring $\mathbb{Q}\left[\mathfrak{S}_{n}\right]$ is $1 / n$. By the inclusion of Sym in FQSym, a ribbon Schur function is mapped to the sum of all permutations with a fixed descent set, depending on its index. For the ribbon Schur function with index $\oslash^{n-1}$, the image is just the permutation Id. For a Lie idempotent in the descent algebra, the coefficient of the ribbon Schur function with index $\oslash^{n-1}$ is therefore $1 / n$.

\subsection{Known series in the dendriform group}

Let us recall some elements of $\mathrm{G}_{\text {Dend }}$ and their properties.

Let $\mathrm{R}$ be the positive sum of all right combs,

$$
R=Y+Y+Y+\ldots
$$

This is the unique solution of the equation

$$
R=Y+Y \prec R \text {. }
$$

Let $\mathrm{L}$ be the alternative sum of all left combs,

$$
\mathrm{L}=-Y+Y-Y+\ldots
$$

This is the unique solution of the equation

$$
\mathrm{L}=-\mathrm{Y}-\mathrm{L} \succ \mathrm{Y} .
$$

The bar involution maps $\mathrm{R}$ to $-\mathrm{L}$. 
Lemma 4.5 The following inversion relation holds:

$$
(1+\mathrm{L}) *(1+\mathrm{R})=1 .
$$

Proof. Both $1+\mathrm{L}$ and $1+\mathrm{R}$ belong to the subalgebra Sym. Indeed $\mathrm{L}$ and $\mathrm{R}$ are the same as

$$
\sum_{k \geq 0}(-1)^{k+1} \oslash^{k} \text { and } \sum_{k \geq 0} \oslash^{k} \text {. }
$$

With the product rule $\mathrm{A} * \mathrm{~B}=\mathrm{A} \oslash \mathrm{B}+\mathrm{A} \oslash \mathrm{B}$ of $\mathbf{S y m}$, this identity is easily proved there. Another proof can be found in [5, Prop. 5.1].

Proposition 4.6 The dendriform image of

$$
\mathrm{CRLS}=\sum_{\mathrm{n} \geq 0} \frac{\mathrm{Crl}_{\mathrm{n}}}{\mathrm{n} !} \quad \text { is } \quad(1+\mathrm{R}) \vee(1+\mathrm{L}) .
$$

Proof. This was proved in [22].

We will now recall and extend some results of [5]. Beware that this article uses slightly different notations.

Recall from section 1.1 that $\operatorname{Lnr}_{\ell}$ is the linear rooted tree with $\ell$ vertices.

Lemma 4.7 The dendriform image of $\Sigma_{\mathrm{T}} \frac{\mathrm{T}}{\operatorname{aut}(\mathrm{T})}$ is given by

$$
(1-\Sigma \mathrm{L}) * \varphi\left(\sum_{\ell \geq 1} \mathrm{Lnr}_{\ell}\right) *(1-\Sigma \mathrm{R}) .
$$

Proof. This follows from [5, Prop. 5.6] (at $\mathrm{q}=\infty)$ and [5, Prop. 6.4]. One also uses Lemma 4.5.

Lemma 4.8 One has

$$
(1-\Sigma \mathrm{L}) * \varphi\left(\sum_{\ell \geq 1} \mathrm{Lnr}_{\ell}\right)=\sum_{n \geq 1} n \mathrm{~L}_{n},
$$

where $\mathrm{L}_{\mathrm{n}}$ is the left comb with $\mathrm{n}$ vertices.

Proof. This is essentially [5, Prop. 5.3].

Lemma 4.9 There holds

$$
\left(\sum_{n \geq 1} n L_{n}\right) *(1-\Sigma R)=(1-\Sigma L) \vee(1-\Sigma R) .
$$

Proof. This is a simple computation in the dendriform algebra, or even in the sub-algebra Sym, with easy cancellations.

Proposition 4.10 The dendriform image of

$$
\mathrm{H}_{1}=\sum_{\mathrm{T}} \frac{\mathrm{T}}{\operatorname{aut}(\mathrm{T})} \quad \text { is } \quad(1-\Sigma \mathrm{L}) \vee(1-\Sigma \mathrm{R}) \text {. }
$$


Proof. This follows from the Lemmas 4.7, 4.8 and 4.9.

Lemma 4.11 In $\mathrm{G}_{\text {Dend }}$, the inverse of $(1-\Sigma \mathrm{L}) \vee(1-\Sigma \mathrm{R})$ is $(1+\mathrm{L}) \vee(1+\mathrm{R})$.

Proof. By lemma 1.1, the inverse of $\mathrm{H}_{1}$ is $\mathrm{H}_{-1}$, which is the suspension of $\mathrm{H}_{1}$.

The result then follows from proposition 4.10, by functoriality of the group construction.

Lemma 4.12 One has

$$
\Sigma \mathrm{R} \circ((1+\mathrm{L}) \vee(1+\mathrm{R}))=-\mathrm{L} .
$$

Proof. By equation (40), it is enough to prove that

$$
\Sigma \mathrm{R} \circ((1+\mathrm{L}) \vee(1+\mathrm{R}))=\mathrm{Y}-(\Sigma \mathrm{R} \circ((1+\mathrm{L}) \vee(1+\mathrm{R}))) \succ Y .
$$

By composition with the inverse of $(1+\mathrm{L}) \vee(1+\mathrm{R})$ given by lemma 4.11, this is equivalent to

$$
\Sigma \mathrm{R}=(1-\Sigma \mathrm{L}) \vee(1-\Sigma \mathrm{R})-\Sigma \mathrm{R} \succ((1-\Sigma \mathrm{L}) \vee(1-\Sigma \mathrm{R})) .
$$

By suspension, this is the same as

$$
\mathrm{R}=(1+\mathrm{L}) \vee(1+\mathrm{R})+\mathrm{R} \succ((1+\mathrm{L}) \vee(1+\mathrm{R})) .
$$

By Lemma 4.5 and (33), this is equivalent to

$$
\mathrm{R}=1 \mathrm{~V}(1+\mathrm{R})
$$

which is just the equation (39).

\section{Series in Sym}

Let $\mathbf{P}_{\mathrm{t}}$ and $\mathbf{N}_{\mathrm{t}}$ be series in variables $\oslash, \oslash$ defined by

$$
\mathbf{P}_{\mathrm{t}}=\sum_{\mathrm{k} \geq 1} \mathbf{c a}_{\mathrm{k}, \mathrm{t}} \oslash^{\mathrm{k}} \text { and } \mathbf{N}_{\mathrm{t}}=\sum_{\mathrm{k} \geq 1}(-1)^{\mathrm{k}} \mathbf{c a}_{\mathrm{k}, \mathrm{t}} \Theta^{\mathrm{k}} \text {, }
$$

where $\mathbf{c a}_{k, t}$ are the $\mathbf{t}$-Narayana fractions defined in $\S 2.9$, and let $\mathbf{P}$ (resp. $\mathbf{N}$ ) be $\mathbf{P}_{\mathrm{t}=0}\left(\right.$ resp. $\left.\mathbf{N}_{\mathrm{t}=0}\right)$.

These series can be considered as ordinary generating series for flows on linear trees, see section 2.9.

Lemma 5.1 One has

$$
\mathbf{P}_{\mathrm{t}}=\frac{1}{1-\mathrm{t}}\left(\emptyset+\mathbf{P}_{\mathrm{t}}\right) \oslash+\frac{\mathrm{b}}{\mathrm{t}}\left(\mathbf{P}_{\mathrm{t}}-\mathbf{P}\right) \oslash
$$

and

$$
\mathbf{N}_{\mathrm{t}}=\frac{-1}{1-\mathrm{t}} \ominus\left(\emptyset+\mathbf{N}_{\mathrm{t}}\right)-\frac{\mathrm{b}}{\mathrm{t}} \ominus\left(\mathbf{N}_{\mathrm{t}}-\mathbf{N}\right) .
$$

Proof. This follows from the fact that the coefficients $\mathbf{c a}_{k, t}$ count flows on linear rooted trees. One has to decompose according to whether the root is an output or not, as already done in the proof of Theorem 2.5. 
One will also need connected variants of $\mathbf{P}$ and $\mathbf{N}$, defined by

$$
\mathbf{P}^{\mathrm{c}}=(\emptyset+\mathrm{b} \mathbf{P}) \oslash \quad \text { and } \quad \mathbf{N}^{\mathrm{c}}=-\oslash(\emptyset+\mathrm{b} \mathbf{N}) .
$$

By (19), these series are generating series for connected closed flows on linear trees. Let us now consider the similar series $\mathbf{P}_{t}^{c}$ for arbitrary connected flows on linear trees.

Every flow on a linear tree can be decomposed as a list of connected components, all but one are closed. One therefore has

$$
\mathbf{P}_{\mathrm{t}}=\mathbf{P}_{\mathrm{t}}^{\mathrm{c}}+\mathbf{P}_{\mathrm{t}} \mathbf{P}^{\mathrm{c}}
$$

A connected flow on a linear tree is either closed, or one can remove one layer of rate on every edge, and obtain any linear flow. This implies that

$$
\mathbf{P}_{\mathrm{t}}^{\mathrm{c}}=\mathbf{P}^{\mathrm{c}}+\mathrm{t} \mathbf{P}_{\mathrm{t}} .
$$

Proposition 5.2 The series $\mathbf{P}^{\mathrm{c}}$ and $\mathbf{N}^{\mathrm{c}}$ satisfy

$$
(1-t) \mathbf{P}_{t}=\mathbf{P}^{c}+\mathbf{P}^{c} \mathbf{P}_{\mathrm{t}} \text { and }(1-\mathrm{t}) \mathbf{N}_{\mathrm{t}}=\mathbf{N}^{\mathrm{c}}+\mathbf{N}_{\mathrm{t}} \mathbf{N}^{\mathrm{c}} .
$$

Proof. It is enough to consider the case of $\mathbf{P}$, by symmetry under the exchange of $\oslash$ and $-\odot$. The equation follows directly from (52) and (53).

Let us now define three series involving both variables $\oslash$ and $\Theta$.

The series $\mathbf{T}$ is defined by

$$
\mathbf{T}=\sum_{k \geq 0} b^{k}(\mathbf{P N})^{k}
$$

and does not depend on the variable $t$.

The series $\mathbf{U}_{t}$ is then defined by

$$
\mathbf{U}_{\mathrm{t}}=(\emptyset+\mathrm{b} \mathbf{N}) \mathbf{T} \mathbf{P}_{\mathrm{t}} .
$$

The first few terms of $\mathbf{U}_{\mathbf{t}}$ are

$$
\mathrm{ca}_{1, \mathrm{t}} \oslash+\mathrm{ca}_{2, \mathrm{t}} \oslash \theta-\mathrm{bca}_{1, \mathrm{t}} \oslash \theta+\ldots
$$

The series $\mathbf{V}_{\mathbf{t}}$ is similarly defined by

$$
\mathbf{V}_{\mathrm{t}}=\mathbf{N}_{\mathrm{t}} \mathbf{T}(\emptyset+\mathrm{b} \mathbf{P}) .
$$

Its first few terms are

$$
-\mathbf{c a}_{1, t} \Theta+\mathbf{c a}_{2, t} \Theta O-\text { b }_{1, t} \Theta O+\ldots
$$

Let $\mathbf{U}($ resp. $\mathbf{V})$ be $\mathbf{U}_{\mathbf{t}=0}\left(\operatorname{resp} . \mathbf{V}_{\mathbf{t}=0}\right)$.

Lemma 5.3 One has

$$
\mathbf{U}_{\mathrm{t}}=\frac{1}{1-\mathrm{t}}\left((\emptyset+\mathrm{b} \mathbf{N}) \mathbf{T}+\mathbf{U}_{\mathrm{t}}\right) \oslash+\frac{\mathrm{b}}{\mathrm{t}}\left(\mathbf{U}_{\mathrm{t}}-\mathbf{U}\right) \oslash
$$

and

$$
\mathbf{V}_{\mathrm{t}}=\frac{-1}{1-\mathrm{t}} \odot\left(\mathbf{T}(\emptyset+\mathrm{b} \mathbf{P})+\mathbf{V}_{\mathrm{t}}\right)-\frac{\mathrm{b}}{\mathrm{t}} \odot\left(\mathbf{V}_{\mathrm{t}}-\mathbf{V}\right)
$$


Proof. This follows from Lemma 5.1 and the definition of $\mathbf{U}_{\mathbf{t}}$ and $\mathbf{V}_{\mathbf{t}}$.

One will need an involution (called the bar involution) on the space of non-commutative formal power series in two variables $\Theta, \oslash$. It is the unique anti-morphism of algebra defined on generators by $\bar{\oslash}=-\Theta$ and $\bar{\Theta}=-\oslash$.

Under the bar involution, $\mathbf{N}_{\mathrm{t}}$ and $\mathbf{P}_{\mathrm{t}}$ are exchanged, $\mathbf{T}$ is fixed and $\mathbf{U}_{\mathrm{t}}$ and $\mathbf{V}_{\mathrm{t}}$ are exchanged.

\section{Series in the dendriform group}

Let $U_{t}$ be the unique dendriform series whose right-completed canopy is given by $\mathbf{U}_{\mathrm{t}}$ :

$$
\mathrm{U}_{\mathrm{t}}=\mathbf{c a}_{1, \mathrm{t}} Y_{+\mathbf{c a}_{2, \mathrm{t}}} Y_{-\mathrm{b}_{\mathrm{ca}}, \mathrm{t}} Y_{+\ldots}
$$

and let $V_{t}$ be the unique dendriform series whose left-completed canopy is given by $\mathbf{V}_{\mathrm{t}}$ :

$$
v_{t}=-\mathbf{c a}_{1, t} Y+\mathbf{c a}_{2, t} Y_{-b_{1, t}} Y_{+\ldots}
$$

It follows from this definition that, under the bar involution on dendriform series, one has $\bar{U}_{t}=-V_{t}$.

Lemma 6.1 Let $\mathrm{u}, \boldsymbol{v}$ be two indeterminates. One has

$$
\left(1+\mathrm{V}_{v}\right) *\left(1+\mathrm{U}_{\mathrm{u}}\right)=1+(v-\mathrm{u}) \mathbf{N}_{v} \mathbf{T} \mathbf{P}_{\mathrm{u}},
$$

where $\mathbf{N}_{\boldsymbol{v}} \mathbf{T} \mathbf{P}_{\mathfrak{u}}$ has to be interpreted as the sum over planar binary trees with the given full canopy.

Proof. This is in fact a computation inside series in $\oslash$ and $\Theta$, by the correspondence between a monomial in $\oslash$ and $\Theta$ and the sum of all planar binary trees having this monomial as their full canopy.

Let us compute $\left(1+\mathrm{V}_{v}\right) *\left(1+\mathrm{U}_{\mathrm{u}}\right)-1$. One finds

$$
\begin{array}{r}
\mathbf{N}_{v} \mathbf{T}(\emptyset+\mathrm{b} \mathbf{P}) \oslash+\oslash(\emptyset+\mathrm{b} \mathbf{N}) \mathbf{T} \mathbf{P}_{\mathrm{u}}+\mathbf{N}_{v} \mathbf{T}(\emptyset+\mathrm{b} \mathbf{P}) \oslash(\emptyset+\mathrm{b} \mathbf{N}) \mathbf{T} \mathbf{P}_{\mathrm{u}} \\
+\mathbf{N}_{v} \mathbf{T}(\emptyset+\mathrm{b} \mathbf{P}) \oslash(\emptyset+\mathrm{b} \mathbf{N}) \mathbf{T} \mathbf{P}_{\mathrm{u}} .
\end{array}
$$

Using the definition (51) of $\mathbf{N}^{c}$ and $\mathbf{P}^{\mathbf{c}}$, one gets

$$
\mathbf{N}_{v} \mathbf{T} \mathbf{P}^{\mathrm{c}}-\mathbf{N}^{\mathrm{c}} \mathbf{T} \mathbf{P}_{\mathrm{u}}+\mathbf{N}_{v} \mathbf{T} \mathbf{P}^{\mathrm{c}}(\emptyset+\mathrm{b} \mathbf{N}) \mathbf{T} \mathbf{P}_{\mathrm{u}}-\mathbf{N}_{v} \mathbf{T}(\emptyset+b \mathbf{P}) \mathbf{N}^{\mathrm{c}} \mathbf{T} \mathbf{P}_{\mathrm{u}} .
$$

Expanding the products, one obtains

$$
\begin{aligned}
\mathbf{N}_{v} \mathbf{T} \mathbf{P}^{\mathrm{c}}-\mathbf{N}^{\mathrm{c}} \mathbf{T} \mathbf{P}_{\mathrm{u}}+\mathbf{N}_{v} \mathbf{T} \mathbf{P}^{\mathrm{c}} \mathbf{T} \mathbf{P}_{\mathrm{u}}+b \mathbf{N}_{v} \mathbf{T} \mathbf{P}^{\mathrm{c}} \mathbf{N} \mathbf{T} \mathbf{P}_{\mathrm{u}} \\
-\mathbf{N}_{v} \mathbf{T N}^{\mathrm{c}} \mathbf{T} \mathbf{P}_{\mathrm{u}}-b \mathbf{N}_{v} \mathbf{T} \mathbf{P} \mathbf{N}^{\mathrm{c}} \mathbf{T} \mathbf{P}_{u} .
\end{aligned}
$$

One can then use the fact that $\mathbf{T}=\emptyset+\mathrm{b} \mathbf{T P N}=\emptyset+\mathrm{b} \mathbf{P N T}$ to split the third and fifth terms, getting

$$
\begin{aligned}
\mathbf{N}_{v} \mathbf{T} \mathbf{P}^{c}-\mathbf{N}^{c} \mathbf{T} \mathbf{P}_{u}+ & \mathbf{N}_{v} \mathbf{T} \mathbf{P}^{c} \mathbf{P}_{u}+b \mathbf{N}_{v} \mathbf{T} \mathbf{P}^{c} \mathbf{P} \mathbf{N} \mathbf{P}_{u}+b \mathbf{N}_{v} \mathbf{T} \mathbf{P}^{c} \mathbf{N} \mathbf{T} \mathbf{P}_{u} \\
& -\mathbf{N}_{v} \mathbf{N}^{c} \mathbf{T} \mathbf{P}_{u}-b \mathbf{N}_{v} \mathbf{T} \mathbf{P} \mathbf{N N}^{c} \mathbf{T} \mathbf{P}_{u}-b \mathbf{N}_{v} \mathbf{T} \mathbf{P} \mathbf{N}^{c} \mathbf{T} \mathbf{P}_{u}
\end{aligned}
$$


Now gathering terms by pairs and using four times the equation (54), one gets, after some cancellations,

$$
(1-u) \mathbf{N}_{v} \mathbf{T} \mathbf{P}_{u}-(1-v) \mathbf{N}_{v} \mathbf{T} \mathbf{P}_{u},
$$

which is the expected result.

\subsection{Flows in the dendriform group}

Let us now consider two series $D_{t}$ and $E_{t}$. Our aim will be to show that they are the respective dendriform images of the series $\mathcal{D}_{t}$ and $\mathcal{E}_{t}$.

The series $D_{t}$ is defined by

$$
D_{t}=\left(1+U_{t}\right) \vee\left(1+V_{t}\right)
$$

and its first few terms are

$$
Y+\operatorname{ca}_{1, t} Y-\operatorname{ca}_{1, t} Y+\ldots
$$

The series $D$ is the value of $D_{t}$ at $t=0$.

The series $E_{t}$ is then defined by

$$
E_{t}=\frac{1}{1-t} D_{t}+\frac{b}{t}\left(D_{t}-D\right)
$$

Its first few terms are

$$
\mathbf{c a}_{1, t} Y+\mathbf{c a}_{2, t} Y_{-\mathbf{c a}_{2, t}} Y_{+\ldots}
$$

The series $E$ is the value of $E_{t}$ at $t=0$.

From these definitions, it results that both $E_{t}$ and $D_{t}$ are fixed under the bar involution of Dend.

Proposition 6.2 One has the following relations

$$
\mathrm{U}_{\mathrm{t}}=\mathrm{R} \circ \mathrm{E}_{\mathrm{t}} \text { and } \mathrm{V}_{\mathrm{t}}=\mathrm{L} \circ \mathrm{E}_{\mathrm{t}} \text {. }
$$

Proof. By symmetry under the bar involution, it is enough to prove the first equation. By the characteristic property (39) of right combs, one just has to show that

$$
\mathrm{U}_{\mathrm{t}}=\mathrm{E}_{\mathrm{t}}+\mathrm{E}_{\mathrm{t}} \prec \mathrm{U}_{\mathrm{t}} .
$$

Let us compute the right hand side using (64). One finds

$$
\frac{1}{1-t} D_{t}+\frac{b}{t}\left(D_{t}-D\right)+\frac{1}{1-t} D_{t} \prec U_{t}+\frac{b}{t}\left(D_{t} \prec U_{t}-D \prec U_{t}\right) .
$$

Using then (63), one gets

$$
\begin{aligned}
& \frac{1}{1-t}\left(1+U_{t}\right) \vee\left(1+V_{t}\right)+\frac{b}{t}\left(\left(1+U_{t}\right) V\left(1+V_{t}\right)-(1+U) V(1+V)\right)+ \\
& \frac{1}{1-t}\left(\left(1+U_{t}\right) V\left(1+V_{t}\right)\right) \prec U_{t}+\frac{b}{t}\left(\left(\left(1+U_{t}\right) V\left(1+V_{t}\right)\right) \prec U_{t}-((1+U) V(1+V)) \prec U_{t}\right),
\end{aligned}
$$


which can be rewritten by (33) as

$$
\begin{array}{r}
\frac{1}{1-\mathrm{t}}\left(1+\mathrm{U}_{\mathrm{t}}\right) \bigvee\left(\left(1+\mathrm{V}_{\mathrm{t}}\right) *\left(1+\mathrm{U}_{\mathrm{t}}\right)\right)+\frac{\mathrm{b}}{\mathrm{t}}\left(\left(1+\mathrm{U}_{\mathrm{t}}\right) \bigvee\left(\left(1+\mathrm{V}_{\mathrm{t}}\right) *\left(1+\mathrm{U}_{\mathrm{t}}\right)\right)\right. \\
-(1+\mathrm{U}) \mathrm{V}\left((1+\mathrm{V}) *\left(1+\mathrm{U}_{\mathrm{t}}\right)\right)
\end{array}
$$

Using Lemma 6.1 , one can replace $\left(1+\mathrm{V}_{\mathrm{t}}\right) *\left(1+\mathrm{U}_{\mathrm{t}}\right)$ by 1 . One obtains

$$
\frac{1}{1-t}\left(1+U_{t}\right) \vee 1+\frac{b}{t}\left(1+U_{t}\right) \vee 1-\frac{b}{t}(1+U) \vee\left((1+V) *\left(1+U_{t}\right)\right)
$$

Using Lemma 6.1 again, one finds

$$
\frac{1}{1-t}\left(1+U_{t}\right) \vee 1+\frac{b}{t}\left(1+U_{t}\right) \vee 1-\frac{b}{t}(1+U) \vee\left(1-t\left(N_{T} T P_{t}\right)\right)
$$

Expanding that, one gets

$$
\frac{1}{1-t} 1 \vee 1+\frac{1}{1-t} U_{t} \vee 1+\frac{b}{t} U_{t} \vee 1-\frac{b}{t} U \vee 1+b 1 \vee\left(N T P_{t}\right)+b U \vee\left(N T P_{t}\right)
$$

We therefore have to show that this expression is simply $\mathrm{U}_{\mathrm{t}}$.

To prove that, let us decompose $U_{t}$ according to the position of the root in the trees. There are four ways to place the root in the full canopy:

- the tree is $Y$, the root can be put between $\Theta$ and $\oslash$,

- the full canopy ends by $\oslash \oslash$, the root can be put between them,

- the full canopy starts by $\bigcirc \mathcal{O}$, the root can be put between them,

- the root can be put after any $\oslash$ followed by $\oslash$ in the full canopy.

Using equation (58), let us describe the first two cases. One gets

- $\frac{1}{1-\mathrm{t}} 1 \vee 1$ for the tree $Y$,

- $\frac{1}{1-\mathrm{t}} \mathrm{U}_{\mathrm{t}} \vee 1+\frac{\mathrm{b}}{\mathrm{t}} \mathrm{U}_{\mathrm{t}} \vee 1-\frac{\mathrm{b}}{\mathrm{t}} \mathrm{U} \vee 1$ for the root between $\oslash$ and $\oslash$.

Using equation (56), let us describe the last two cases. One gets

- $\mathrm{b} 1 \vee\left(\mathbf{N T} \mathbf{P}_{\mathrm{t}}\right)$ for the root between $Q$ and $Q$,

- $\mathrm{bU} \vee\left(\mathbf{N T} \mathbf{P}_{\mathrm{t}}\right)$ for the root between $\oslash$ and $\Theta$.

Note that the last case is slightly more subtle, as the cut takes places inside the $\mathbf{T}$ factor and one has to use the expression (63) for the series $\mathbf{T}$.

It follows that (66) is exactly the expansion of $U_{t}$ according to the possible positions of the root in the canopy.

Corollary 6.3 One has

$$
D_{t}=\left((1+R) \circ E_{t}\right) \vee\left((1+L) \circ E_{t}\right) .
$$

Proof. This follows from (63) and Proposition 6.2. 
This is readily reformulated by lemma 4.2 using the $\diamond$ operation as

$$
D_{t}=((1+R) \vee(1+L)) \diamond\left(Y, E_{t}\right) .
$$

Theorem 6.4 The dendriform images of $\varepsilon_{t}$ and $\mathcal{D}_{t}$ are $E_{t}$ and $D_{t}$.

Proof. The series $\mathcal{E}_{\mathrm{t}}$ and $\mathcal{D}_{\mathrm{t}}$ are characterized by the equations (23) and (22). By Proposition 4.6 and results of appendix A, the dendriform image of (22) is exactly (68). The dendriform image of (23) is exactly (64). Therefore $E_{t}$ and $D_{t}$ satisfy equations that characterize the dendriform images of $\varepsilon_{t}$ and $\mathcal{D}_{t}$, and the statement follows.

\subsection{Explicit product formulas for coefficients}

The equations (56) and (57) provide an explicit description of the coefficients of the series $U_{t}$ and $V_{t}$.

More precisely, the coefficient of a planar binary tree $\tau$ in the series $U_{t}$ can be found as follows. One considers the right-completed canopy of $\tau$ (including the rightmost leaf but not the leftmost leaf). It admits a unique coarsest decomposition into blocks of the shape $\oslash^{k}$ and $\oslash^{\ell}$ for $k, \ell \geq 1$. Every block of length $\ell$ in this decomposition contributes a Narayana factor $\mathbf{c a}_{\ell}$, but the rightmost block contributes instead a $\mathbf{t}$-Narayana factor $\mathbf{c a}_{\ell, \mathrm{t}}$. There is an additional factor of $\mathrm{b}$ to the power the number of $\odot$ blocks and $(-1)$ to the power the number of Q.

For example, the coefficient of the leftmost planar binary tree of figure 6 , whose right-completed canopy is 000000 , is

$$
\mathrm{b}^{2} \mathbf{c a} \mathbf{a}_{1} \mathbf{c a} \mathbf{c a}_{1} \mathbf{c a _ { 2 , \mathrm { t } }} \text {. }
$$

There is a similar description for $V_{t}$. One considers the left-completed canopy of $\tau$ (including the leftmost leaf but not the rightmost leaf) and decompose it into maximal blocks of $\oslash$ and $\Theta$. Every such block of length $\ell$ contributes a Narayana factor $\mathbf{c a}_{\ell}$, but the leftmost block contributes instead a $\mathrm{t}$-Narayana factor $\mathbf{c a}_{\ell, \mathrm{t}}$. There is an additional factor of $\mathrm{b}$ to the power the number of $\oslash$ blocks and $(-1)$ to the power the number of $\Theta$.

One can also interpret the definition (63) as giving the explicit coefficients of the series $D_{t}$.

More precisely, the coefficient of a planar binary tree $\tau$ in the series $D_{t}$ can be found as follows. Consider the canopy of $\tau$, and cut it into two parts according to the position of the root of tree. Decompose both parts into maximal blocks of $\oslash$ and $\oslash$. Every such block of length $\ell$ contributes a Narayana factor $\mathbf{c a}_{\ell}$, but the two blocks that are closest to the root contributes instead a t-Narayana factor $\mathbf{c a}_{\ell, \mathbf{t}}$. There is an additional factor of $\mathrm{b}$ to the power the number of $\odot$ blocks in the left part plus the number of $\oslash$ blocks in the right part, and $(-1)$ to the power the number of $Q$.

For example, the coefficient of the leftmost planar binary tree of figure 6 , whose canopy is cut into 000 and 00 , is

$$
\mathrm{b}^{2} \mathrm{ca}_{1} \mathbf{c a} \mathbf{a}_{2, \mathrm{t}} \mathbf{c a}_{1, \mathrm{t}} \mathbf{c a} \mathbf{a}_{1} \text {. }
$$


Letting $t=0$ in this description, one observes that the coefficient of a tree in $\mathrm{D}$ depends only on its canopy. This is obvious for the factors associated with blocks and for the sign. As for the power of $b$, it can be described as the number of $\odot$ blocks in the canopy, excluding the last (rightmost) block.

It follows that $D$ is in the descent algebra. Moreover, this description of $D_{n}$ is exactly the value at $a=1$ of the description given in [20, Th. 10.1] and we therefore recover this theorem. Let us give its statement here.

Corollary 6.5 The homogeneous components $\mathrm{D}_{\mathrm{n}}$ are Lie idempotents and satisfy

$$
D_{n} \cdot D_{n}=n c a_{n-1} D_{n},
$$

in the symmetric group ring of $\mathfrak{S}_{n}$.

To determine the precise constant of proportionality, one uses Lemma 4.4 and the fact that the coefficient of the ribbon Schur function $\oslash^{n-1}$ is $\mathbf{c a}_{n-1}$.

One can now use (64) to give an explicit description of the coefficients of the series $E_{t}$.

As $D_{t}$ and $D$ have all but two of their factors in common, all these factors are also in $E_{t}$. The remaining factor is

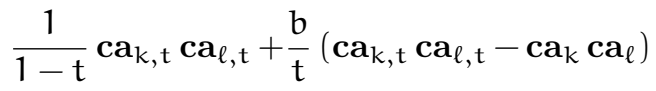

which is the fraction counting flows on the rooted trees $B_{+}\left(\operatorname{Ln} r_{k}, \operatorname{Ln} r_{\ell}\right)$.

Therefore, the coefficient of a planar binary tree $\tau$ in the series $E_{t}$ can be found as follows. Consider the canopy of $\tau$, and cut it into two parts according to the position of the root of tree. Decompose both parts into maximal blocks of $\oslash$ and $\odot$, excluding the two central blocks. Every such block of length $\ell$ contributes a Narayana factor $\mathbf{c a} \ell$. The two central blocks together are of the shape $\oslash^{k} \oslash^{\ell}$. We associate with this the coefficient of the rooted tree $B_{+}\left(\operatorname{Ln} r_{k}, \operatorname{Ln} r_{\ell}\right)$ in the series $\mathcal{E}_{\mathrm{t}}$.

There is an additional factor of $\mathrm{b}$ to the power the number of $\odot$ blocks in the left part plus the number of $\oslash$ blocks in the right part, and $(-1)$ to the power the number of $\odot$.

\subsection{Connected flows in the dendriform group}

Let us now introduce the dendriform image $\mathrm{E}_{\mathrm{t}}^{\mathrm{c}}$ of the series $\mathcal{E}_{\mathrm{t}}^{\mathrm{c}}$ of connected flows. As $\mathcal{E}_{\mathrm{t}}^{\mathrm{c}}$ is related to $\mathcal{E}_{\mathrm{t}}$ by equation (26), one gets, by using Proposition 4.10 and results of appendix $A$, that $E_{t}^{c}$ is defined by

$$
\mathrm{E}_{\mathrm{t}}=((1-\Sigma \mathrm{L}) \vee(1-\Sigma \mathrm{R})) \diamond\left(\mathrm{E}_{\mathrm{t}}^{\mathrm{c}}, \mathrm{E}^{\mathrm{c}}\right) \text {. }
$$

Letting $t=0$, one gets

$$
E=((1-\Sigma L) \vee(1-\Sigma R)) \circ E^{c} .
$$

Proposition 6.6 The series $\mathrm{E}_{\mathrm{t}}^{\mathrm{c}}$ admits the following expression

$$
\begin{aligned}
\frac{1}{1-t} Y+\left(\frac{t}{1-t}+b\right) 1 \vee \mathbf{N}_{t} \mathbf{T P} & -\left(\frac{t}{1-t}+b\right) \mathbf{N T} \mathbf{P}_{t} \vee 1 \\
& -t\left(\frac{t}{1-t}+b\right) \mathbf{N} \mathbf{T P}_{t} \vee \mathbf{N}_{t} \mathbf{T P}
\end{aligned}
$$


Proof. Using lemma 4.11, one can invert the relation (72) between $E_{\text {and }} E^{\mathfrak{c}}$ as

$$
((1+\mathrm{L}) \vee(1+\mathrm{R})) \circ \mathrm{E}=\mathrm{E}^{\mathrm{c}} .
$$

On the other hand, using lemma 4.2, lemma 4.3 and lemma 4.5, one can invert the relation (71) between $E_{t}$ and $E_{t}^{c}$ to obtain

$$
E_{t}^{c}=\left(1-\Sigma R \circ E^{c}\right) V_{E_{t}}\left(1-\Sigma L \circ E^{c}\right) .
$$

But by (74) and Lemma 4.12, one deduces that

$$
\Sigma \mathrm{R} \circ \mathrm{E}^{\mathrm{c}}=\Sigma \mathrm{R} \circ((1+\mathrm{L}) \vee(1+\mathrm{R})) \circ \mathrm{E}=-\mathrm{L} \circ \mathrm{E},
$$

and by symmetry that

$$
\Sigma \mathrm{L} \circ \mathrm{E}^{\mathrm{c}}=\mathrm{L} \circ((1+\mathrm{L}) \vee(1+\mathrm{R})) \circ \mathrm{E}=-\mathrm{R} \circ \mathrm{E} .
$$

One therefore gets using prop. 6.2 that

$$
E_{t}^{c}=(1+L \circ E) V_{E_{t}}(1+R \circ E)=(1+V) V_{E_{t}}(1+U)
$$

By the equation (64), one finds

$$
\frac{1}{1-t}(1+V) \bigvee_{D_{t}}(1+U)+\frac{b}{t}\left((1+V) V_{D_{t}}(1+U)-(1+V) V_{D}(1+U)\right)
$$

Using then the definition (63) of $D_{t}$, one gets

$$
\left(\frac{1}{1-t}+\frac{b}{t}\right)(1+V) *\left(1+U_{t}\right) V\left(1+V_{t}\right) *(1+U)-\frac{b}{t}(1+V) *(1+U) V(1+V) *(1+U)
$$

By Lemma 6.1, one gets

$$
\left(\frac{1}{1-t}+\frac{b}{t}\right)\left(1-t \mathbf{N T P}_{t}\right) \vee\left(1+t \mathbf{N}_{t} \mathbf{T P}\right)-\frac{b}{t} 1 \vee 1
$$

This gives the expected result, after simplification.

Recall from (28) that one can write

$$
\mathrm{E}^{\mathrm{c}}=\mathrm{Y}+\mathrm{bF} / \mathrm{Y}-\mathrm{b} Y \backslash \mathrm{F},
$$

where $F$ is the dendriform image of the series $\mathcal{F}$ introduced in (28).

Corollary 6.7 The series $\mathrm{E}^{\mathrm{c}}$ admits the following expression

$$
Y+b 1 \vee N T P-b N T P \vee 1 .
$$

The series $\mathrm{F}$ is given by

$$
-\mathbf{N T P}
$$

and belongs to the descent algebra. 
One can use (77) to give an explicit description of the coefficients of F.

More precisely, let $\tau$ be a planar binary tree. Then consider the full canopy of $\tau$, and its coarsest decomposition into blocks of the shape $\oslash^{k}$ or $\oslash^{\ell}$ for $k, \ell \geq 1$. To each such block of size $\ell$, one associate a factor $\mathbf{c a}_{\ell}$.

Then the coefficient of $\tau$ is the product of these factors, times a power of $\mathrm{b}$ given by the number of $\odot$ blocks minus 1 and times $(-1)$ to the power the number of $\odot$ minus 1 .

For example, the coefficient of the leftmost planar binary tree of figure 6 , whose full canopy is 0000000 , is

\section{b ca $\mathrm{ca}_{2} \mathrm{ca}_{1} \mathrm{ca}_{2}$.}

Using Prop. 6.6, one can also give a description of the coefficients of the series $\mathrm{E}_{\mathrm{t}}^{\mathrm{c}}$ of connected flows.

Let us consider a planar binary tree $\tau$ with at least 2 inner vertices. One considers the full canopy of $\tau$, and cut it into two parts by using the position of the root. One distinguish three cases: the root can either be placed between ( and $\Theta$ at the left of of the full canopy, or between $\oslash$ and $\oslash$ at the right of the full canopy, or between $\oslash$ and $\odot$ inside the full canopy. In each case, one can translate the corresponding term in (73) into a description of the factors of the coefficient of $\tau$ in $\mathrm{E}_{\mathrm{t}}^{\mathrm{c}}$.

The series $\mathrm{F}$ provides new Lie idempotents.

Proposition 6.8 The homogeneous component $\mathrm{F}_{\mathrm{n}}$ of the series $\mathrm{F}$ satisfies

$$
F_{n} \cdot F_{n}=n c a_{n} F_{n}
$$

in the symmetric group ring of $\mathfrak{S}_{\mathfrak{n}}$.

The constant $\mathbf{n} \mathbf{c a}_{\mathrm{n}}$ is determined by Lemma 4.4, using that the coefficient of the ribbon Schur function $\oslash^{n-1}$ (corresponding to the full canopy $\oslash^{n}$ ) is $\mathbf{c a}_{n}$.

Let us consider now the series $F_{t}=-(1-t) \mathbf{N}_{t} \mathbf{T} \mathbf{P}_{t}$, which gives back $F$ when $t=0$.

Assuming that Conjecture 2.14 holds, one can introduce a global series $\mathcal{F}_{\mathrm{t}}$ and propose the following conjecture.

Conjecture 6.9 The series $\mathrm{F}_{\mathrm{t}}$ is the dendriform image of the series $\mathcal{F}_{\mathrm{t}}$.

If this is true, then the series $F_{t}$ provides new Lie idempotents.

Conjecture 6.10 The homogeneous component $\mathrm{F}_{\mathrm{n}, \mathrm{t}}$ of the series $\mathrm{F}_{\mathrm{t}}$ satisfies

$$
F_{n, t} \cdot F_{n, t}=n c a_{n, t} F_{n, t}
$$

in the symmetric group ring of $\mathfrak{S}_{\mathfrak{n}}$.

The constant $\mathbf{n} \mathbf{c a}_{\mathbf{n}, \mathrm{t}}$ in this conjecture is given by Lemma 4.4, using that the coefficient of the ribbon Schur function $\oslash^{n-1}$ (corresponding to the full canopy $\oslash \oslash^{\mathrm{n}}$ ) is $\mathbf{c a}_{\mathrm{n}, \mathrm{t}}$.

Conjecture 6.10 has been checked up to $\mathfrak{S}_{6}$ included. 


\subsection{Description of $Z$}

Let $\mathbf{Z}$ be the dendriform image of $\boldsymbol{Z}$, introduced in (30). We propose here a conjectural description of the coefficients of $\mathbf{Z}$.

For positive integers $p$ and $q$, let us define polynomials

$$
z_{p, q}=\sum_{k \geq 0}\left(\begin{array}{l}
p \\
k
\end{array}\right)\left(\begin{array}{l}
q \\
k
\end{array}\right) b^{p+q+1-k}
$$

If $p=q$, this polynomial is essentially a Narayana polynomial of type B.

Let now $\tau$ be a planar binary tree of size $n$. Consider the full canopy of $\tau$ and decompose it into blocks of the shape $\Theta^{p} \oslash^{q}$ with $p, q \geq 1$. To each such block $\oslash^{\mathfrak{p}} \oslash^{\mathrm{q}}$, one associates a factor $(-1)^{p-1} z_{p-1, q-1}$.

The coefficient of $\tau$ in the series $z$ seems to be the product of these factors associated with blocks, divided by $b$. The total degree with respect to $b$ is $n$ minus the number of blocks.

If this description holds, the coefficient of $\tau$ would depend only on its canopy. This would imply the following result.

Conjecture 6.11 The homogeneous component $\mathrm{Z}_{\mathrm{n}}$ of the series $\mathrm{Z}$ is in the descent algebra and satisfies

$$
\mathrm{Z}_{\mathrm{n}} \cdot \mathrm{Z}_{\mathrm{n}}=n \mathrm{~b}^{\mathrm{n}-1} \mathrm{Z}_{\mathrm{n}}
$$

in the symmetric group ring of $\mathfrak{S}_{\mathrm{n}}$.

Note that one uses Lemma 4.4 to get this precise statement.

Question 6.12 Are the zeroes of the polynomials $z_{\mathrm{p}, \mathrm{q}}$ real?

It is known that the generalized Narayana numbers associated with finite Coxeter groups have only real roots, see [21, §5.2].

Remark 6.13 The polynomials $z_{\mathrm{p}, \mathrm{p}+1}$ and $z_{\mathrm{p}, \mathrm{p}}$, as well as the polynomials $\mathcal{F}$ for forks seem to appear in the article [14], which deals with symmetric functions. The relationship with the present work is not clear to us.

\section{A Appendix}

We present here a general setting for the combinatorial use of some algebraic structures related to operads with specific properties. The reader may like to keep in mind that the Pre-Lie and dendriform operads are the motivating examples.

Recall that a species is a functor from the category of finite sets and bijections to the category of finite sets. For more on the notion of species, the reader may want to consult the book [3]. 


\section{A.1 Rooted-operads}

Let $\mathcal{P}$ be a species, such that $\mathbb{Z} \mathcal{P}$ is endowed with an operad structure (in the category of $\mathbb{Z}$-modules).

We assume that $\mathcal{P}$ comes with a morphism of species to the species of pointed sets. This means that to every $\mathcal{P}$-structure $T$ on a finite set $\mathrm{I}$, one associates an element of I. We will call this element the root of $\mathrm{T}$.

We assume also that the composition $o_{i}$ is compatible with the root in the following sense:

- if $i$ is the root of $S$, the root of every term of $S \circ_{i} T$ is the root of $T$,

- otherwise the root of every term of $S \circ_{i} T$ is the root of $S$.

We will call this structure a rooted-operad.

Examples of this situation are provided by the Pre-Lie operad, the Dendriform operad, the NAP operad [15] and the Perm operad. For the Pre-Lie and NAP operad, the underlying species is the species of rooted trees, and one takes the root of each tree. For the Dendriform operad, the underlying species can be described as (rooted) planar binary trees with labels on internal vertices, and one also takes the root of each tree. For Perm, the underlying species is the species of pointed sets, and the root morphism is the identity.

Let $\mathcal{P}$ and $\mathcal{P}^{\prime}$ be two rooted-operads. A morphism of rooted-operads $\theta$ from $\mathcal{P}$ to $\mathcal{P}^{\prime}$ is a morphism of operads from $\mathbb{Z} \mathcal{P}$ to $\mathbb{Z} \mathcal{P}^{\prime}$ such that for every element $p$ of $\mathcal{P}$, the root of every term of $\theta(p)$ is the root of $p$.

Lemma A.1 Let $\theta$ be a morphism of operads from $\mathbb{Z} \mathcal{P}$ to $\mathbb{Z} \mathcal{P}^{\prime}$ given by its value on elements of $\mathcal{P}$ that are generators of $\mathbb{Z} \mathcal{P}$. If , for every generator $p$, the root of every term in $\theta(\mathrm{p})$ is the root of $\mathrm{p}$, then $\theta$ is a morphism of rooted-operads.

Proof. Let us prove by induction on the arity of $p \in \mathcal{P}$ that every term in $\theta(p)$ has the same root as $p$. This is clearly true for the unit of $\mathbb{Z} \mathcal{P}$.

Let $x$ be an element of $\mathcal{P}$. If $x$ is a generator, then the statement is true by hypothesis.

Otherwise, $x$ can be written as a linear combination

$$
x=\sum_{\alpha} \lambda_{\alpha} p_{\alpha} \circ_{i_{\alpha}} q_{\alpha}
$$

where $p_{\alpha}$ and $q_{\alpha}$ are elements of $\mathcal{P}$ of smaller arities. Because $\mathcal{P}$ is a rootedoperad, every composition $p_{\alpha} \circ_{i_{\alpha}} q_{\alpha}$ is a linear composition of terms sharing the same root. One can therefore remove in the sum above every $\alpha$ such that $p_{\alpha} \circ_{i_{\alpha}} q_{\alpha}$ does not have the same root as $x$. Then one has

$$
\theta(x)=\sum_{\alpha} \lambda_{\alpha} \theta\left(p_{\alpha}\right) \circ_{i_{\alpha}} \theta\left(q_{\alpha}\right)
$$

and every term $\theta\left(p_{\alpha}\right) \circ_{i_{\alpha}} \theta\left(q_{\alpha}\right)$ has the same root as $\chi$. The induction step is done.

This condition holds for the morphism $\varphi$ from the Pre-Lie operad to the Dendriform operad, which is therefore a morphism of rooted-operads. 


\section{A.2 Triple operation associated with rooted-operads}

Let $\mathcal{P}$ be a rooted-operad, as defined in the previous section.

Let us associate with $\mathcal{P}$ the coinvariant space $\mathbb{Z} \mathcal{P}_{\mathfrak{S}}$, which is the free module over $\mathbb{Z}$ with basis indexed by isomorphism classes of $\mathcal{P}$-structures on all finite sets. One can also describe it as

$$
\mathbb{Z} \mathcal{P}_{\mathfrak{S}}=\oplus_{\mathrm{m} \geq 1} \mathbb{Z} \mathcal{P}(\mathrm{m})_{\mathfrak{S}}
$$

where $\mathcal{P}(m)$ is $\mathcal{P}(\{1,2, \ldots, m\})$. We will call $[\mathrm{T}]$ the basis element associated with the isomorphism class of the $\mathcal{P}$-structure $\mathrm{T}$.

On $\mathbb{Z} \mathcal{P}_{\mathfrak{S}}$, there is a natural structure of monoid, whose associative product is defined using the composition of the operad $\mathcal{P}$, see for example [5, App. A]. Let us recall this construction and introduce a refinement of it, which uses the existence of the root.

Let $s=\sum_{m} s_{m}, t=\sum_{m} t_{m}$ be elements of $\mathbb{Z} \mathcal{P}_{\mathfrak{S}}$, with $s_{m}, t_{m}$ elements of $\mathbb{Z} \mathcal{P}(m)_{\mathfrak{S}}$. Choose any representatives $x_{m}, y_{m}$ of $s_{m}, t_{m}$ in $\mathbb{Z} \mathcal{P}(m)$.

The monoid structure is given by

$$
s \circ t=\sum_{m \geq 1} \sum_{n_{1}, \ldots, n_{m} \geq 1}\left\langle x_{m}\left(y_{n_{1}}, \ldots, y_{n_{m}}\right)\right\rangle,
$$

where \langle\rangle is the quotient map to the coinvariant space, and $\left(x, y_{1}, \ldots, y_{k}\right) \mapsto$ $x\left(y_{1}, \ldots, y_{k}\right)$ is the global composition map of the operad $\mathcal{P}$, here using the numbering of the parts to match inputs of $x$ with the $y$ 's.

Let now $s=\sum_{m} s_{m}, t=\sum_{m} t_{m}$ and $u=\sum_{m} u_{m}$ be elements of $\mathbb{Z} \mathcal{P}_{\mathfrak{S}}$, with $s_{m}, t_{m}, u_{m}$ elements of $\mathbb{Z} \mathcal{P}(m)_{\mathfrak{S}}$. Choose representatives $x_{m}, y_{m}$ and $z_{m}$ of $s_{m}, t_{m}, u_{m}$ in $\mathbb{Z} \mathcal{P}(m)$, such that the root of $x_{m}$ is 1 .

Let us introduce the following operation

$$
s \diamond(t, u)=\sum_{m \geq 1} \sum_{n_{1}, \ldots, n_{m} \geq 1}\left\langle x_{m}\left(y_{n_{1}}, z_{n_{2}}, \ldots, z_{n_{m}}\right)\right\rangle .
$$

This is well defined, because $\left\langle x_{m}\left(y_{n_{1}}, z_{n_{2}}, \ldots, z_{n_{m}}\right)\right\rangle$ does not depend on the chosen representatives, provided that the root of $x_{m}$ is 1 .

Proposition A.2 The operation $(\mathrm{s}, \mathrm{t}, \mathrm{u}) \mapsto \mathrm{s} \diamond(\mathrm{t}, \mathrm{u})$ satisfies

$$
(s \diamond(t, u)) \diamond(v, w)=s \diamond(t \diamond(v, w), u \circ w),
$$

and is linear with respect to $\mathrm{s}$ and to $\mathrm{t}$. When $\mathrm{t}=\mathrm{u}$, it reduces to the monoid structure:

$$
s \diamond(t, t)=s \circ t .
$$

The unit 1 of the operad gives a unit [1], i.e. one has

$$
[1] \diamond(\mathrm{t}, \mathrm{u})=\mathrm{t} \quad \text { and } \quad \mathrm{s} \diamond([1],[1])=\mathrm{s} .
$$

Proof. The linearity with respect to the parameters $s$ and $t$, the special case when $t=u$ and the unit properties all follows by inspection from the definition (84).

Concerning formula (85), one has to compute both sides by choosing representatives with care. It is necessary to choose the representatives for $s$ and for $t$ such that their root is 1 . Then the result follows from the usual axioms of operads, and from the conditions on roots imposed by the definition of rootedoperads. 
One could call this kind of structure a rooted-monoid. There is an obvious notion of morphism of rooted-monoids.

Proposition A.3 The construction that maps a rooted-operad $\mathcal{P}$ to the space $\mathbb{Z} \mathcal{P}_{\mathfrak{S}}$ endowed with the operation $\diamond$ is a functor from the category of rootedoperads to the category of rooted-monoids.

Proof. By the definition of the morphisms of rooted operads, the image of an element with root 1 is a sum of terms with root 1 . The functoriality then follows by inspection of the definition (84).

\section{A.3 Combinatorial use: rooted case}

Let us now present the combinatorial application of the operation $\diamond$ that is used in the main part of the article.

Let $\mathcal{P}$ be a species, such that $\mathbb{N} \mathcal{P}$ is endowed with an rooted-operad structure (in the category of $\mathbb{N}$-modules rather than $\mathbb{Z}$-modules).

Examples of this situation are also provided by the Pre-Lie operad, the Dendriform operad, the NAP operad and the Perm operad.

Let $X$ be a species with a morphism of species to $\mathcal{P}$. When an $X$-structure $\alpha$ has image the $\mathcal{P}$-structure $S$, we will say that $\alpha$ is over $S$. The set of these structures will be denoted by $X_{/ s}$. Its cardinality only depends on the isomorphism class of $S$. Let $s_{X}$ be the generating series

$$
s_{X}=\sum_{[S]} \# X / s \frac{[S]}{\operatorname{aut} S},
$$

where the sum runs over the set of isomorphism classes of $\mathcal{P}$-structures, and $\operatorname{aut}(S)$ is the cardinal of the automorphism group of any representative $S$ of $[S]$. This is an element of $\mathbb{Q} \mathcal{P}_{\mathfrak{S}}$.

Let I be a finite set. Let $\mathrm{U}$ be a $\mathcal{P}$-structure on I. Let $\pi$ be a partition of I. Let $S$ be a $\mathcal{P}$-structure on the set of parts of $\pi$, and let $\left(T_{e}\right)_{e}$ be $\mathcal{P}$-structures on the parts $e$ of $\pi$. One will denote by $\mathbf{f}_{S,\left(T_{e}\right)_{e}}^{\mathrm{U}}$ the coefficient of $\mathrm{U}$ in the global composition $S\left(\left(T_{e}\right)_{e}\right)$ in the operad $\mathcal{P}$. This is a positive integer by the assumption that $\mathbb{N} \mathcal{P}$ is an operad.

Let us consider now four species A, B, C and D, each one with a morphism of species to $\mathcal{P}$.

Suppose that (hypothesis $H_{b}(A, B, C, D)$ ) for any finite set $I$, any $r \in I$ and any $\mathcal{P}$-structure $\mathrm{U}$ on $\mathrm{I}$ with root $\mathrm{r}$, there is a bijection between

- the set $\mathrm{D} / \mathrm{u}$ of $\mathrm{D}$-structures over $\mathrm{U}$,

- the set of tuples

$$
\left(\pi, S,\left(T_{e}\right)_{e}, \alpha,\left(\beta_{e}\right)_{e}, \lambda\right),
$$

where $\pi$ is a partition of $I$ with a part $\epsilon$ containing $r, S$ is a $\mathcal{P}$-structure with root $\epsilon$ on the set of parts of $\pi,\left(T_{e}\right)_{e}$ are $\mathcal{P}$-structures on the parts $e$ of $\pi$ such that the root of $T_{\epsilon}$ is $r, \alpha \in A / s, \beta_{\epsilon} \in B / T_{\epsilon}, \beta_{e} \in C_{/ T_{e}}$ for $e \neq \epsilon$ and $\lambda \in\left\{1, \ldots, f_{S,\left(T_{e}\right)_{e}}^{\mathrm{U}}\right\}$.

Proposition A.4 Under the hypothesis $\mathrm{H}_{\natural}(\mathrm{A}, \mathrm{B}, \mathrm{C}, \mathrm{D})$, one has

$$
s_{A} \diamond\left(s_{B}, s_{C}\right)=s_{D} \text {. }
$$


Proof. Let us fix an integer $m \geq 1$ and an integer $r \in\{1, \ldots, m\}$.

From the hypothesis $H_{\natural}(A, B, C, D)$, one can obtain the following equality

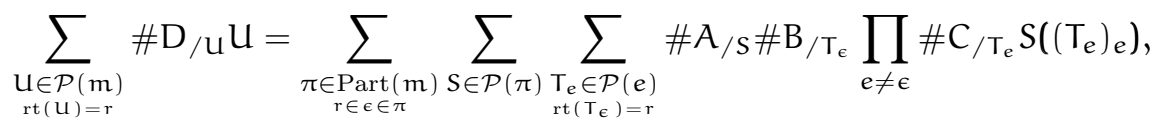

where $\operatorname{Part}(m)$ is the set of partitions of $\{1, \ldots, m\}$.

Let us take the image of this equality by the projection to coinvariants.

The left hand side becomes

$$
\sum_{[\mathrm{U}] \in \mathcal{P}(\mathrm{m})_{\mathfrak{S}}} \frac{(\mathrm{m}-1) ! \# \mathrm{D} / \mathrm{u}}{\operatorname{aut} \mathrm{U}}[\mathrm{U}] .
$$

The right hand side becomes

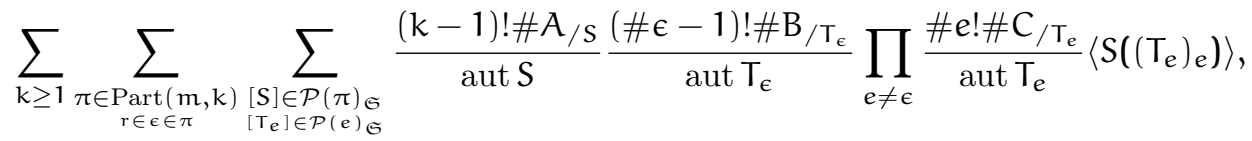

where $\operatorname{Part}(m, k)$ is the set of partitions of $\{1, \ldots, m\}$ into $k$ parts.

By using the factor $(k-1)$ ! to define an order on the parts of the partition, such that the first part contains the root, one gets

$$
\sum_{k \geq 1} \sum_{\substack{\pi_{1}, \ldots, \pi_{k} \\ r \in \pi_{1}}} \sum_{\substack{[S] \in \mathcal{P}(k) \\\left[T_{i}\right] \in \mathcal{P}\left(\pi_{i}\right)_{\mathfrak{S}}}} \frac{\# A / S}{\operatorname{aut} S} \frac{\left(\# \pi_{1}-1\right) ! \# B / T_{1}}{\operatorname{aut~} T_{1}} \prod_{j=2}^{k} \frac{\# \pi_{j} ! \# C_{/ T_{j}}}{\operatorname{aut~} T_{j}}\left\langle S\left(\left(T_{j}\right)_{j}\right)\right\rangle .
$$

By using the multinomial formula for the number of ordered partitions of $m$ into $k$ parts of size $n_{1}, \ldots, n_{k}$, one gets

$$
(m-1) ! \sum_{k \geq 1} \sum_{\substack{n_{1}, \ldots, n_{k} \geq 1 \\ n_{1}+\cdots+n_{k}=m}} \sum_{\substack{[S] \in \mathcal{P}(k)_{\mathfrak{T}} \\\left[T_{i} \in \mathcal{P}\left(n_{i}\right)_{\mathcal{G}}\right.}} \frac{\# A / S}{\operatorname{aut} S} \frac{\# B / T_{1}}{\operatorname{aut~} T_{1}} \prod_{j=2}^{k} \frac{\# C / T_{j}}{\operatorname{aut~} T_{j}}\left\langle S\left(T_{1},\left(T_{j}\right)_{j \geq 2}\right)\right\rangle .
$$

From the equality between (90) and (91), one deduces the result, after division by $(m-1)$ ! and summation over $m \geq 1$.

If $\mathrm{B}=\mathrm{C}$, the operation $\diamond$ reduces to the monoid product $\mathrm{o}$, and the hypothesis $\mathrm{H}_{\mathfrak{t}}(A, B, B, D)$ can be formulated without using the root. One can obtain in this way a simpler analog of proposition A.4 valid for any operad on a species $\mathcal{P}$. This is made explicit in the next paragraph.

\section{A.4 Combinatorial use: group case}

Let $\mathcal{P}$ be a species, such that $\mathbb{N} \mathcal{P}$ is endowed with an operad structure.

Let us consider now three species $A, B$ and $C$, each one with a morphism of species to $\mathcal{P}$.

Suppose that (hypothesis $H_{\sharp}(A, B, C)$ ) for any finite set I and any $\mathcal{P}$ structure $\mathrm{U}$ on $\mathrm{I}$, there is a bijection between

- the set $\mathrm{C}_{/ \mathrm{U}}$ of $\mathrm{C}$-structures over $\mathrm{U}$, 
- the set of tuples

$$
\left(\pi, S,\left(T_{e}\right)_{e}, \alpha,\left(\beta_{e}\right)_{e}, \lambda\right),
$$

where $\pi$ is a partition of I, S is a $\mathcal{P}$-structure on the set of parts of $\pi$, $\left(\mathrm{T}_{e}\right)_{e}$ are $\mathcal{P}$-structures on the parts $e$ of $\pi, \alpha \in A / s, \beta_{e} \in \mathrm{B}_{/ \mathrm{T}_{e}}$ and $\lambda \in\left\{1, \ldots, \mathbf{f}_{\mathrm{S},\left(\mathrm{T}_{e}\right)_{e}}^{\mathrm{U}}\right\}$.

Proposition A.5 Under the hypothesis $\mathrm{H}_{\sharp}(\mathrm{A}, \mathrm{B}, \mathrm{C})$, one has

$$
s_{\mathrm{A}} \circ \mathrm{s}_{\mathrm{B}}=\mathrm{s}_{\mathrm{C}} \text {. }
$$

\section{References}

[1] J.-C. Arditti. Dénombrement des arborescences dont le graphe de comparabilité est hamiltonien. Discrete Math., 5:189-200, 1973.

[2] J.-C. Arditti and R. Cori. Hamilton circuits in the comparability graph of a tree. In Combinatorial theory and its applications, I (Proc. Colloq., Balatonfüred, 1969), pages 41-53. North-Holland, Amsterdam, 1970.

[3] F. Bergeron, G. Labelle, and P. Leroux. Combinatorial species and tree-like structures, volume 67 of Encyclopedia of Mathematics and its Applications. Cambridge University Press, Cambridge, 1998. Translated from the 1994 French original by Margaret Readdy, With a foreword by Gian-Carlo Rota.

[4] F. Chapoton. The anticyclic operad of moulds. Int. Math. Res. Not. IMRN, (20):Art. ID rnm078, 36, 2007

[5] F. Chapoton. A rooted-trees q-series lifting a one-parameter family of Lie idempotents. Algebra Number Theory, 3(6):611-636, 2009.

[6] F. Chapoton and M. Livernet. Pre-Lie algebras and the rooted trees operad. Internat. Math. Res. Notices, (8):395-408, 2001.

[7] F. Chapoton and M. Livernet. Relating two Hopf algebras built from an operad. Int. Math. Res. Not. IMRN, (24):Art. ID rnm131, 27, 2007.

[8] G. Duchamp, F. Hivert, and J.-Y. Thibon. Noncommutative symmetric functions. VI. Free quasi-symmetric functions and related algebras. Internat. J. Algebra Comput., 12(5):671-717, 2002.

[9] J. Ecalle. A tale of three structures: the arithmetics of multizetas, the analysis of singularities, the Lie algebra ARI. In Differential equations and the Stokes phenomenon, pages 89-146. World Sci. Publ., River Edge, NJ, 2002.

[10] J. Ecalle. Multizetas, perinomal numbers, arithmetical dimorphy, and ARI/GARI. Ann. Fac. Sci. Toulouse Math. (6), 13(4):683-708, 2004.

[11] J. Ecalle and F. Menous. Well-behaved convolution averages and the nonaccumulation theorem for limit-cycles. In The Stokes phenomenon and Hilbert's 16th problem (Groningen, 1995), pages 71-101. World Sci. Publ., River Edge, NJ, 1996. 
[12] I. M. Gelfand, D. Krob, A. Lascoux, B. Leclerc, V. S. Retakh, and J.-Y. Thibon. Noncommutative symmetric functions. Adv. Math., 112(2):218348, 1995.

[13] V. P. Kostov, A. Martínez-Finkelshtein, and B. Z. Shapiro. Narayana numbers and Schur-Szegö composition. J. Approx. Theory, 161(2):464-476, 2009.

[14] M. Lassalle. Narayana polynomials and hall-littlewood symmetric functions, 2011.

[15] M. Livernet. A rigidity theorem for pre-Lie algebras. J. Pure Appl. Algebra, 207(1):1-18, 2006.

[16] J.-L. Loday, A. Frabetti, F. Chapoton, and F. Goichot. Dialgebras and related operads, volume 1763 of Lecture Notes in Mathematics. SpringerVerlag, Berlin, 2001.

[17] J.-L. Loday and M. O. Ronco. Hopf algebra of the planar binary trees. Adv. Math., 139(2):293-309, 1998.

[18] C. Malvenuto and C. Reutenauer. Duality between quasi-symmetric functions and the Solomon descent algebra. J. Algebra, 177(3):967-982, 1995.

[19] F. Menous. Les bonnes moyennes uniformisantes et une application à la resommation réelle. Ann. Fac. Sci. Toulouse Math. (6), 8(4):579-628, 1999.

[20] F. Menous, J.-C. Novelli, and J.-Y. Thibon. Mould calculus, polyhedral cones and characters of combinatorial Hopf algebras. 2011.

[21] V. Reiner and V. Welker. On the Charney-Davis and Neggers-Stanley conjectures. J. Combin. Theory Ser. A, 109(2):247-280, 2005.

[22] M. O. Ronco. Primitive elements in a free dendriform algebra. In New trends in Hopf algebra theory (La Falda, 1999), volume 267 of Contemp. Math., pages 245-263. Amer. Math. Soc., Providence, RI, 2000.

[23] M. Schocker. Über die höheren Lie-Darstellungen der symmetrischen Gruppen. Bayreuth. Math. Schr., (63):103-263, 2001.

[24] J.-Y. Thibon. Lectures on noncommutative symmetric functions. In Interaction of combinatorics and representation theory, volume 11 of MSJ Mem., pages 39-94. Math. Soc. Japan, Tokyo, 2001. 